\title{
Aryl Hydrocarbon Receptor (AhR) Activation by 2,3,7,8-Tetrachlorodibenzo- $p$-Dioxin (TCDD) Dose-Dependently Shifts the Gut Microbiome Consistent with the Progression of Non-Alcoholic Fatty Liver Disease
}

\author{
Russell R. Fling ${ }^{1,2}$ and Timothy R. Zacharewski ${ }^{2,3, *}$ \\ 1 Department of Microbiology and Molecular Genetics, Michigan State University, \\ East Lansing, MI 48824, USA; flingrus@msu.edu \\ 2 Institute for Integrative Toxicology, Michigan State University, East Lansing, MI 48824, USA \\ 3 Department of Biochemistry and Molecular Biology, Michigan State University, East Lansing, MI 48824, USA \\ * Correspondence: tzachare@msu.edu; Tel.: +1-(517)-355-1607
}

\section{check for} updates

Citation: Fling, R.R.; Zacharewski, T.R. Aryl Hydrocarbon Receptor (AhR) Activation by 2,3,7,8Tetrachlorodibenzo- $p$-Dioxin (TCDD) Dose-Dependently Shifts the Gut Microbiome Consistent with the Progression of Non-Alcoholic Fatty Liver Disease. Int. J. Mol. Sci. 2021, 22, 12431. https://doi.org/10.3390/ ijms222212431

Academic Editor: Walter Wahli

Received: 30 October 2021

Accepted: 16 November 2021

Published: 18 November 2021

Publisher's Note: MDPI stays neutral with regard to jurisdictional claims in published maps and institutional affiliations.

Copyright: (c) 2021 by the authors. Licensee MDPI, Basel, Switzerland. This article is an open access article distributed under the terms and conditions of the Creative Commons Attribution (CC BY) license (https:/ / creativecommons.org/licenses/by/ $4.0 /)$.

\begin{abstract}
Gut dysbiosis with disrupted enterohepatic bile acid metabolism is commonly associated with non-alcoholic fatty liver disease (NAFLD) and recapitulated in a NAFLD-phenotype elicited by 2,3,7,8-tetrachlorodibenzo- $p$-dioxin (TCDD) in mice. TCDD induces hepatic fat accumulation and increases levels of secondary bile acids, including taurolithocholic acid and deoxycholic acid (microbial modified bile acids involved in host bile acid regulation signaling pathways). To investigate the effects of TCDD on the gut microbiota, the cecum contents of male C57BL/ 6 mice orally gavaged with sesame oil vehicle or $0.3,3$, or $30 \mu \mathrm{g} / \mathrm{kg}$ TCDD were examined using shotgun metagenomic sequencing. Taxonomic analysis identified dose-dependent increases in Lactobacillus species (i.e., Lactobacillus reuteri). Increased species were also associated with dose-dependent increases in bile salt hydrolase sequences, responsible for deconjugation reactions in secondary bile acid metabolism. Increased $L$. reuteri levels were further associated with mevalonate-dependent isopentenyl diphosphate (IPP) biosynthesis and o-succinylbenzoate synthase, a menaquinone biosynthesis associated gene. Analysis of the gut microbiomes from cirrhosis patients identified an increased abundance of genes from the mevalonate-dependent IPP biosynthesis as well as several other menaquinone biosynthesis genes, including o-succinylbenzoate synthase. These results extend the association of lactobacilli with the AhR/intestinal axis in NAFLD progression and highlight the similarities between TCDD-elicited phenotypes in mice to human NAFLD.
\end{abstract}

Keywords: 2,3,7,8-tetrachlorodibenzo-p-dioxin; dioxin; aryl hydrocarbon receptor; non-alcoholic fatty liver disease; gut microbiome; fibrosis; gut dysbiosis; secondary bile acids

\section{Introduction}

Non-alcoholic fatty liver disease (NAFLD) is estimated to affect $25 \%$ of the global population and is defined as a spectrum of progressive pathologies, including steatosis, immune cell infiltration/inflammation, fibrosis, and cirrhosis. It is associated with increased risk for hepatocellular carcinoma and is the second leading cause of liver transplants in the USA [1]. Other pathologies, including obesity, type 2 diabetes (T2D), and coronary heart disease, demonstrate a high co-occurrence with NAFLD, e.g., $~ 40-70 \%$ in T2D patients and $\sim 90 \%$ in obese patients [2]. A multi-hit hypothesis for NAFLD proposes several contributing factors to development and progression, including disruptions in the immune system, adipose tissue metabolism, and the gut microbiome [3].

Emerging evidence also suggests that environmental contaminants may play an underappreciated role in gut dysbiosis and NAFLD development [4-11]. Specifically, 2,3,7,8-tetrachlorodibenzo-p-dioxin (TCDD), a persistent environmental organochloride pollutant, induces steatosis and the progression to steatohepatitis with fibrosis in mice 
resembling human NAFLD development [9,12-14]. TCDD-induced dyslipidemia also exhibits other similar NAFLD characteristics, such as decreased VLDL secretion, free fatty acid accumulation, inhibition of $\beta$-oxidation, and disrupted cholesterol and bile acid metabolism $[9,12,15-17]$.

The effects of TCDD and other related polychlorinated dibenzo-p-dioxins (PCDDs), dibenzofurans (PCDFs), and coplanar biphenyls (PCBs) as well as polyaromatic hydrocarbons (PAHs), are mediated through activation of the aryl hydrocarbon receptor (AhR), a basic helix-loop-helix/Per-Arnt-Sim transcription factor typically associated with xenobiotic metabolism [18]. In addition, the AhR plays an essential role in gut homeostasis through regulation of the immune system and bile acid metabolism $[9,12,19,20]$ with endogenous and xenobiotic AhR ligands affecting the gut microbiome congruent with NAFLD-like pathology [8-10]. Moreover, gut dysbiosis is commonly reported in NAFLD, making the gut microbiome an attractive target for non-invasive diagnostic tools and a potential target for intervention [21,22].

Although the AhR exhibits promiscuous binding activity for a wide variety of structurally diverse xenobiotics, natural products, and endogenous metabolites, its endogenous role remains unknown [23]. Upon ligand binding, the cytosolic AhR translocates to the nucleus where it disassociates from its chaperone proteins and dimerizes with the AhR nuclear transporter (ARNT). The AhR/ARNT heterodimer complex then binds to dioxin response elements located throughout the genome, affecting gene expression [24].

Endobiotic ligands for the AhR include host-derived metabolites, such as tryptophan catabolites (e.g., L-kynurenine), microbial-produced indole derivatives (e.g., indole-3aldehyde produced by Lactobacillus reuteri), and compounds derived from fruits and cruciferous vegetables (e.g., indole-3-carbinol [ICZ]) [23]. Microbially produced indoles activate $A h R$ in the intestine, affecting barrier function and homeostasis by regulating the intestinal immune system through $\mathrm{CD}^{+} \mathrm{T}$-cell differentiation and the induction of interleukin (IL)-22 and IL-10 cytokine production [24]. AhR-dependent IL-22 induction subsequently increases antimicrobial peptide expression in intestinal epithelial cells, thus, inhibiting pathogen infection and inflammation [24-26].

Knockout models and/or treatment with endogenous and xenobiotic AhR ligands results in shifts in the gut microbiome with diverse effects depending on the model and ligand $[8,11,26-28]$. Shifts in the Firmicutes/Bacteroidetes ratio can differ between AhR ligands, e.g., 2,3,7,8-tetrachlorodibenzofuran decreased the ratio [8] whereas TCDD increased it [11].

However, responses in various AhR models are in agreement regarding increased secondary bile acids $[8,9]$ and effects on segmented filamentous bacteria $[8,11,27]$. AhR knockout models, and treatment with TCDD or other endogenous compounds also demonstrate strong correlations between AhR activation and enrichment of Lactobacillus species, i.e., L. reuteri [26-30]. Tryptophan catabolism to AhR ligands by Lactobacillus species is a proposed mechanism for gut microbial regulation of AhR signaling that modulates intestinal and gut microbiome homeostasis [26].

Bile acids also affect the gut microbiome by exerting antimicrobial activity [31]. Conversely, the gut microbiota play critical roles in host bile acid homeostasis through microbial metabolism that qualitatively and quantitatively impact bile acid composition with consequences for bile acid activated signaling pathways in the host. The gut microbiome performs the first step of bile acid deconjugation with subsequent oxidation, reduction, or dehydroxylation reactions to produce diverse secondary bile acid molecular species [32].

Select secondary bile acids, e.g., glycodeoxycholic acid [GDCA], demonstrate higher inhibition of bacterial growth compared to other primary and secondary bile acids [33]. In regard to the host, some secondary bile acids, e.g., lithocholic acid [LCA] and deoxycholic acid [DCA]), exhibit a high affinity for the farnesoid $x$ receptor (FXR) and $G$ proteincoupled bile acid receptor (TGR5, a.k.a., GPBAR1), which regulate glucose, lipid, and bile acid homeostasis [34-36]. In human NAFLD, the secondary bile acid metabolism is 
disrupted with bile acid analogs that target the FXR and TGR5 signaling pathways under development for the treatment of liver disease [22,31,37].

Previous work demonstrated that the serum levels of LCA and DCA increased following TCDD treatment suggesting enrichment for the microbial bile acid metabolism [9]. To further explore dose-dependent disruptions in the gut microbiome and microbial metabolism relevant to the progression of NAFLD-like pathologies, shotgun metagenomic analysis was used to examine the dose dependent taxonomic and metabolic disruptions elicited by TCDD.

\section{Results}

\subsection{TCDD-Elicited Toxicity Enriched for Lactobacillus Species}

Taxonomic analysis identified significant dose-dependent population shifts among caecum microbiota in response to TCDD. While no significance was observed between treatment groups at the phylum level, a decreasing trend was observed for Bacteroidetes concurrent with increasing trends in Firmicutes abundance (Figure 1A).

A

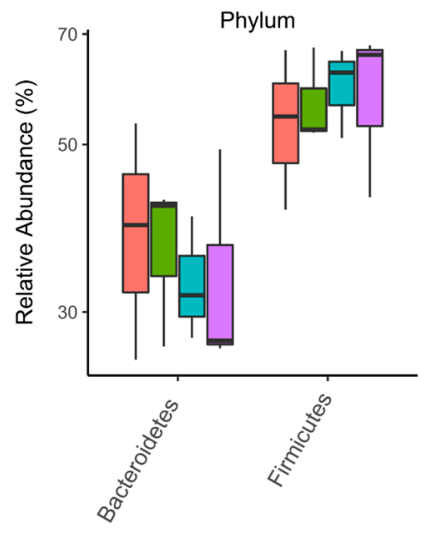

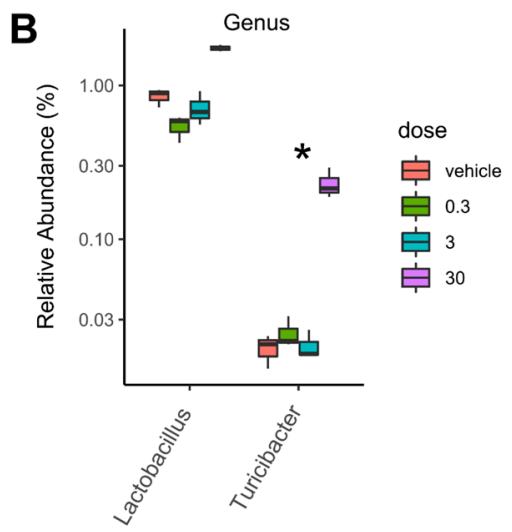

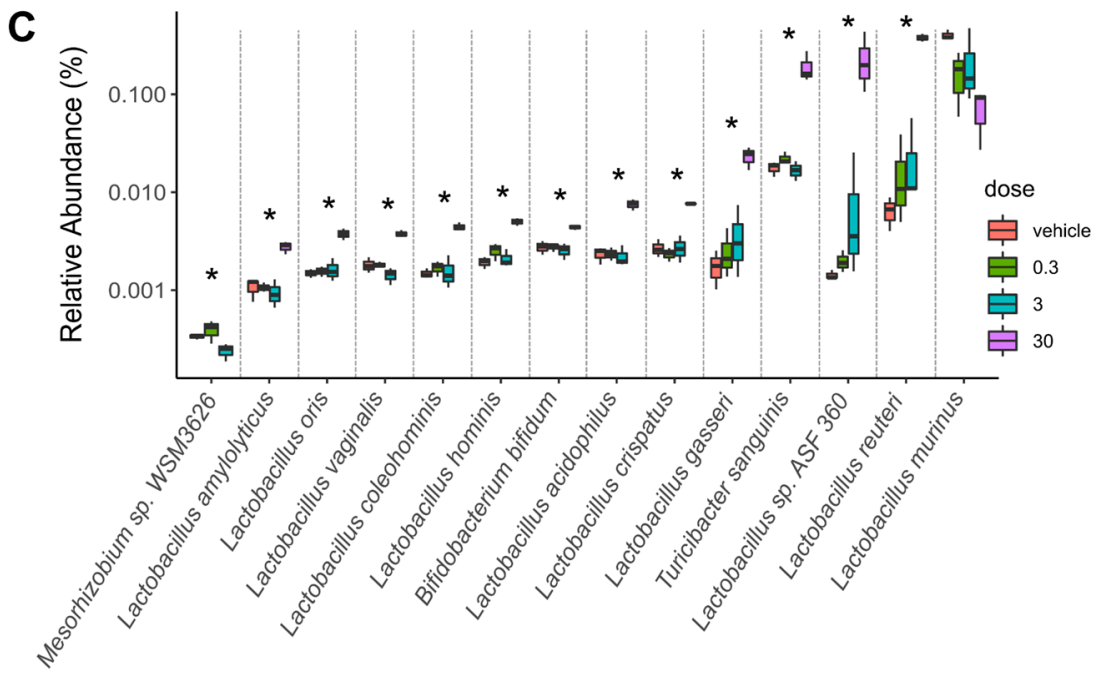

Species

Figure 1. TCDD enriched Lactobacillus species in the cecum microbiota. Taxa abundance were assessed in metagenomic cecum samples from male C57BL/ 6 mice following oral gavage with sesame oil vehicle or $0.3,3$, or $30 \mu \mathrm{g} / \mathrm{kg}$ TCDD every 4 days for 28 days $(n=3)$. Significant shifts in relative abundances of taxa are presented at the (A) phylum, (B) genus, (C) and species levels. Significance is denoted with an asterisk $\left({ }^{*}\right.$; adjusted $p$-value $\left.<0.1\right)$.

At the genus level, Turicibacter was enriched by TCDD while the genus Lactobacillus trended towards enrichment (Figure 1B). Interestingly, at the species level, 10 out of 13 enriched species were from the Lactobacillus genus (e.g., L. reuteri and Lactobacillus sp. 
ASF360) as well as Turicibacter sanguinis. Conversely, the most abundant Lactobacillus species in vehicle treated mice, Lactobacillus murinus, trended towards a dose-dependent decrease (Figure 1C). The major changes in taxa were observed in the $30 \mu \mathrm{g} / \mathrm{kg}$ TCDD treatment group.

\subsection{Bile Salt Hydrolase (Bsh) Levels Correlated with Significantly Enriched Species}

Many Lactobacillus species deconjugate primary conjugated bile acids mediated by bile salt hydrolases (BSH), imparting bile acid tolerance [38]. To further investigate the effect of TCDD on bile acid metabolism, $b$ sh sequences were annotated and quantified within metagenomic samples. Annotations to $b s h$ were increased by TCDD and associated with enriched species, including L. reuteri and T. sanguinis (Figures 1C and 2A, and Table S1).

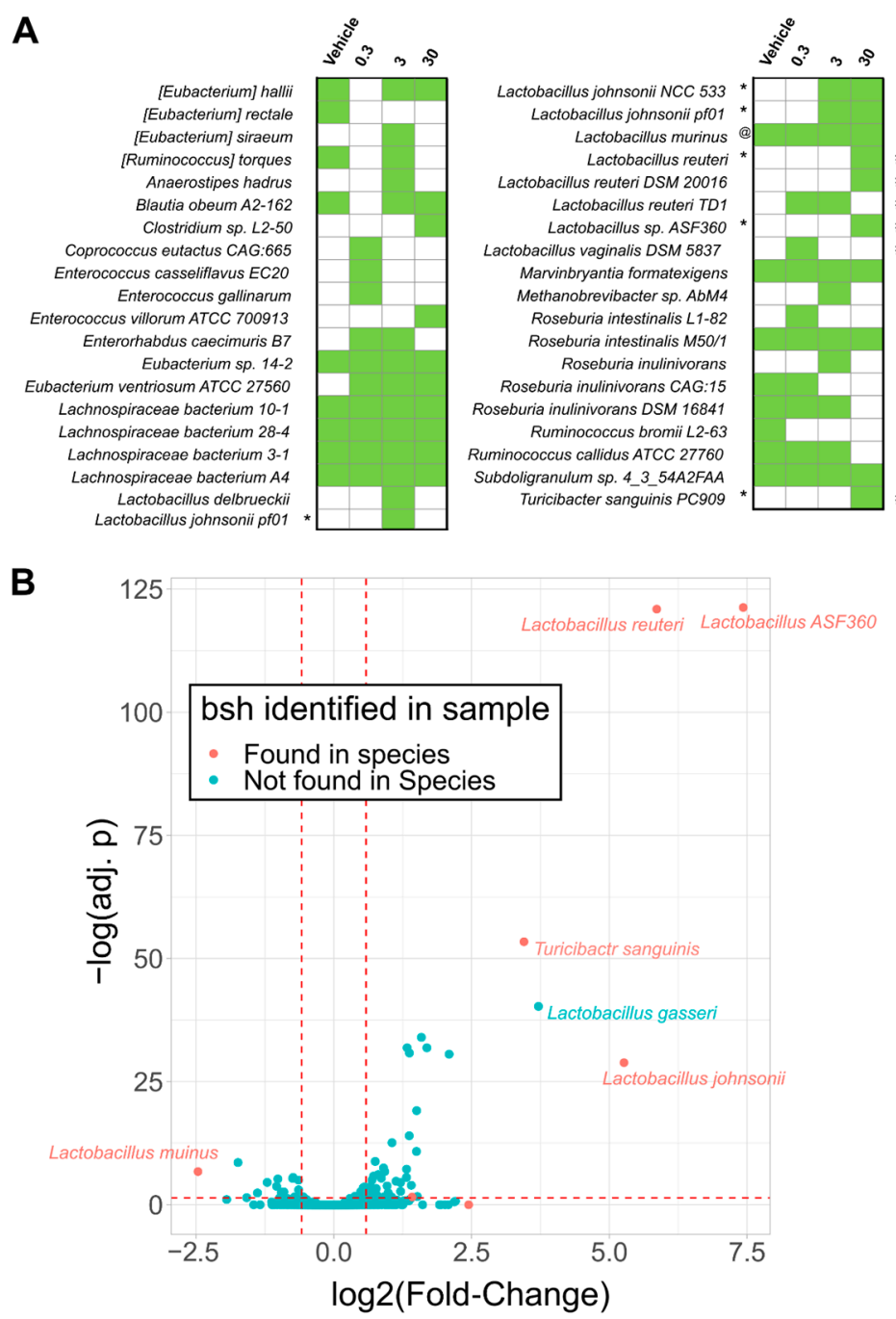

Figure 2. TCDD enriched Lactobacillus species possessing bile salt hydrolase $(b s h)$. The presence of $b s h$ gene sequences were assessed in metagenomic caecum samples from male C57BL/6 mice following oral gavage with sesame oil vehicle or $0.3,3$, or $30 \mu \mathrm{g} / \mathrm{kg}$ TCDD every 4 days for 28 days using three independent cohorts $(n=3)$. (A) The presence (green boxes) or absence of $b s h$ sequences detected in any of the metagenomic samples $(n=3)$ are denoted within the respective treatment groups. Significant increases $\left(^{*}\right)$ or decreases (@) in normalized bsh abundances (adj. $\left.p<0.1\right)$ are denoted. Also denoted is significantly increased species (\#) determined by taxonomic analysis that 
corresponded with respective RefSeq species $b s h$ annotations. Significance was determined by Maaslin2 R package. (B) Volcano plot displaying log2(fold-changes) in relative abundance of species between vehicle and $30 \mu \mathrm{g} / \mathrm{kg}$ TCDD treatment groups versus - $\log$ (adjusted $p$-values [adj. $p$ ]). Red dots denotes $b s h$ sequences detected in $30 \mu \mathrm{g} / \mathrm{kg}$ TCDD treatment group. Significance was determined by the DeSeq2 R package comparing only vehicle and $30 \mu \mathrm{g} / \mathrm{kg}$ TCDD groups. Red dashed lines are reference to $-\log (0.05)$ value for the $y$-axis and -1 and 1 for the $x$-axis.

Conversely, L. murinus associated bsh annotations exhibited a dose-dependent decrease consistent with decreasing trends in taxonomic abundance. Although not reaching significance, many $b s h$ sequences were also associated with unclassified Lachnospiraceae species, including Lachnospiraceae bacterium A4, a community member reaching 5-23\% relative abundance in the cecum metagenomic samples (Figure 2A). In contrast, Lactobacillus gasseri was enriched but no bsh sequences were identified (Figure 2B). To summarize, the top enriched species were also associated with increased abundances in $b s h$ levels in the cecum.

\subsection{TCDD Enriched for Mevalonate-Dependent Isoprenoid Biosynthesis}

To investigate other metabolic pathways imparting competitive advantages to TCDDelicited gut environmental stresses, functional gene annotations associated with $L$. reuteri, the highest enriched species, were assessed. Among enriched uniref 90 annotations in the cecum metagenomic dataset was the aromatic amino acid aminotransferase (UniRef90 A0A2S1ENB9) also classified to L. reuteri (Table S2). Aromatic amino acid aminotransferase produces a tryptophan metabolite, indole-3-aldehyde, a known AhR ligand reported to induce IL-22 in vivo [26]. Among 39 enzyme commission (EC) annotations that were enriched and associated with $L$. reuteri were several annotated to the isoprenoid biosynthesis pathway (Figure 3, Figures S1 and S3).

Bacteria biosynthesize the isoprenoid, isopentenyl diphosphate (IPP), either through the mevalonate-dependent pathway, which is also found in mammals, or the methylerythritol phosphate (MEP)-pathway. Both L. reuteri and Lactobacillus johnsonii were the major contributors to mevalonate-dependent IPP biosynthesis pathway enrichment with almost all genes in the pathway increased by TCDD; four out of six of the genes significantly increased by TCDD (Figure 3 and Figure S1). Gene enrichment in the alternative MEPpathway were unchanged by TCDD. For L. murinus, only two EC annotations (EC 2.7.1.148, 4-diphosphocytidyl-2-C-methyl-D-erythritol (CDP-ME) kinase, and EC 5.3.3.2, isopentenyldiphosphate Delta-isomerase) were identified in the MEP pathway also found in L. reuteri (Figure S2).

HUMAnN 3.0 analysis of a published metagenomics dataset of fecal samples from human cirrhotic patients (https:/ / www.ncbi.nlm.nih.gov/bioproject/PRJEB6337/, accessed on 25 March 2021) [39] revealed strikingly similar results to our caecum samples from TCDD treated mice. Specifically, increased gene abundance associated with the mevalonatedependent pathways was also evident in patients with compensated and decompensated liver disease (Figure 4). 

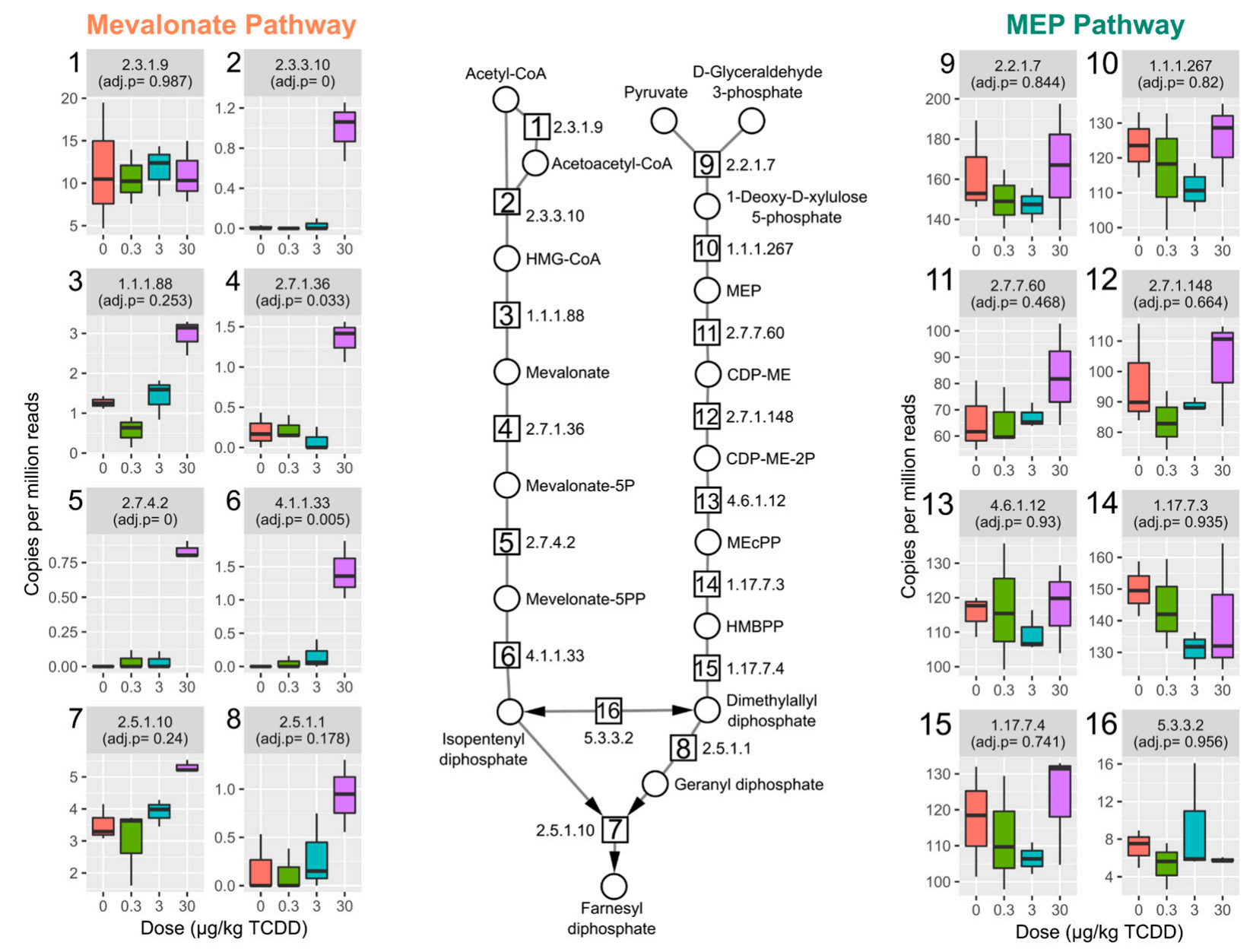

Figure 3. TCDD enriched genes from the mevalonate-dependent isoprenoid biosynthesis pathway. Relative abundance of genes involved in isoprenoid biosynthesis and grouped by enzyme commission (EC) numbers for the mevalonate dependent and 2-C-methyl-D-erythritol 4-phosphate (MEP) pathways in cecum samples from male C57BL6 mice following oral gavage with sesame oil vehicle or $0.3,3$, or $30 \mu \mathrm{g} / \mathrm{kg}$ TCDD every 4 days for 28 days $(n=3)$. Individual box plots are also numbered with the EC number matching the enzymatic step in pathway schematic. Adjusted $p$-values (adj. $p$ ) were determined by the Maaslin2 R package. Abbreviations: 3-hydroxyl-3-methyl-clutaryl-CoA (HMG-CoA), (R)-5-Phosphomevalonate (mevalonate-5P), (R)-5-Diphosphomevalonate (mevalonate-5PP), 2-C-Methyl-D-erythritol 4-phosphate (MEP), 4-(Cytidine 5'-diphospho)-2-C-methyl-D-erythritol (CDP-ME), 4-(Cytidine 5'-diphospho)-2-C-methyl-D-erythritol (DEP-ME-2P), 2-CMethyl-D-erythritol 2,4-cyclodiphosphate (MEcPP), and 1-Hydroxy-2-methyl-2-butenyl 4-diphosphate (HMBPP).

Compensated cirrhosis is defined as no decrease in liver function while decompensated cirrhosis exhibits decreased liver function. Among decompensated patients with cirrhosis, the mevalonate dependent IPP pathway was increased in 7 out of 8 EC numbers required for de novo IPP biosynthesis (Figure 4). Taxa annotated to genes in the pathway exhibited a wide variety in genera for each EC number in human samples compared to murine cecum samples from this study (Figure S3). Taxonomy classified to a majority of the mevalonate-dependent genes were from the Lactobacillaceae family, including Enterococcus, Lactobacillus, Streptococcus genera (Figure S3 and Table S5). Lactobacillus and Streptococcus species, including L. reuteri and Streptococcus anginosus, a known pathogen in liver abscesses [40], were among species classified to the pathway (Tables S4 and S5). 
Mevalonate Pathway
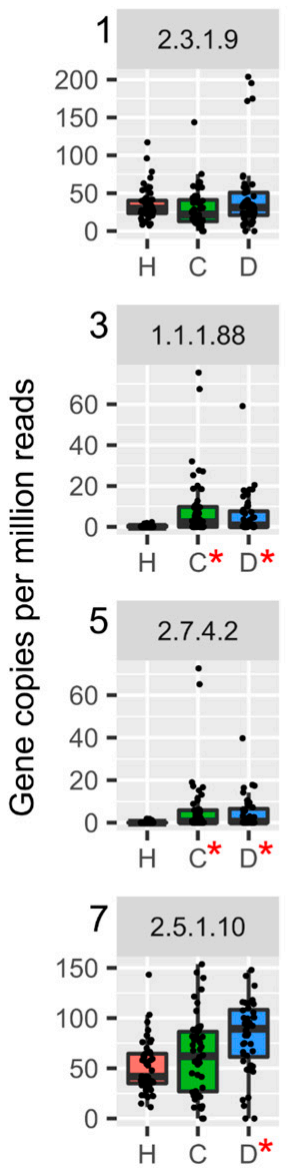
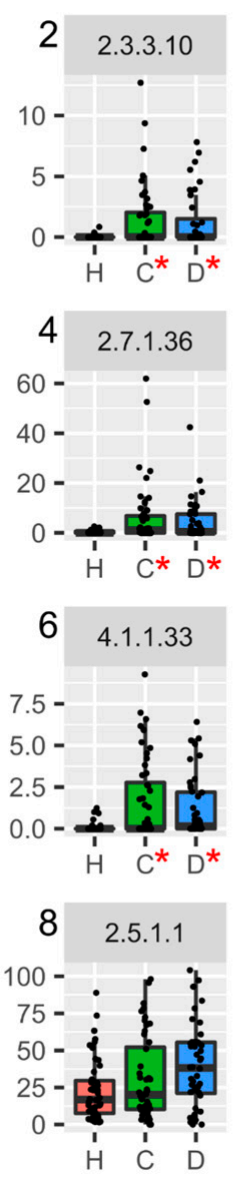
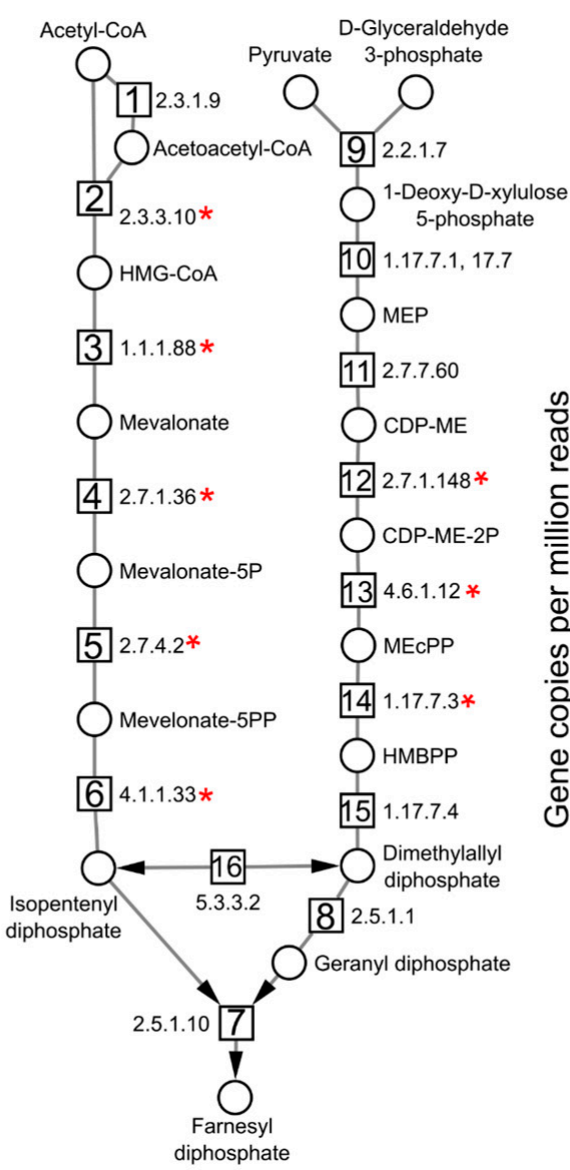

MEP Pathway
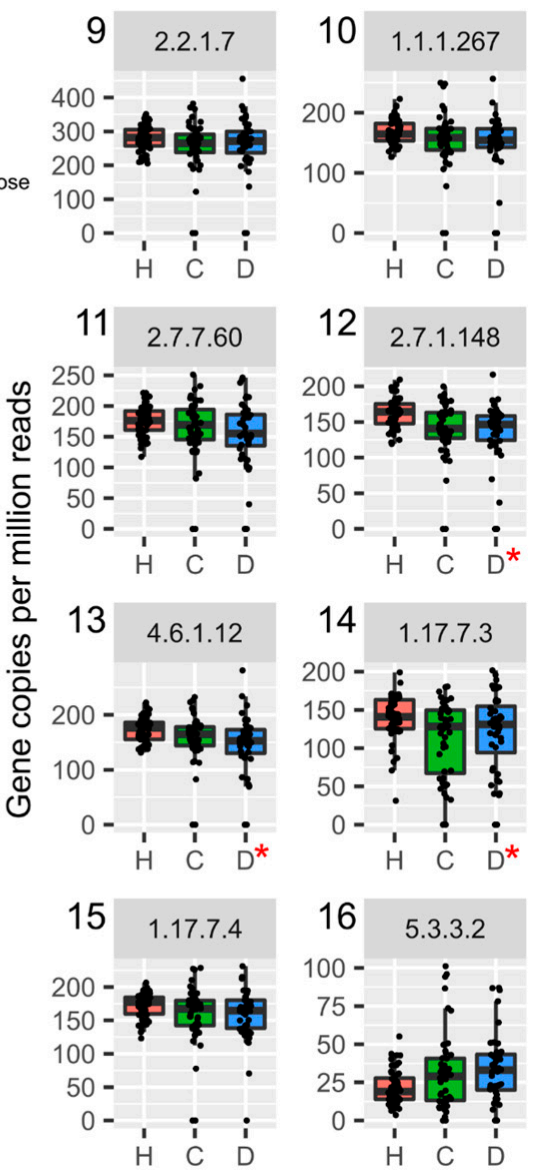

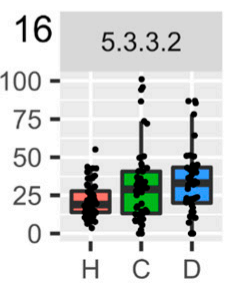

Figure 4. Mevalonate-dependent isoprenoid biosynthesis genes are enriched in a published metagenomics dataset of fecal samples from cirrhosis patients. Humann3 analysis of fecal gut microbiomes in healthy $(\mathrm{H}, \mathrm{red}, n=52)$, compensated (C, green, $n=48$ ), or decompensated ( $\mathrm{D}$, blue, $n=44$ ) cirrhosis for mevalonate-dependent and methyl-D-erythritol 4-phosphate (MEP) pathways. Individual boxplots are numbered with the EC number matching the enzymatic step in pathway schematic. Significance is denoted with a red asterisk $(*$, adjusted $p$-values $<0.05)$ compared to healthy group. Abbreviations.: 3hydroxyl-3-methyl-clutaryl-CoA (HMG-CoA), (R)-5-Phosphomevalonate (mevalonate-5P), (R)-5-Diphosphomevalonate (mevalonate-5PP), 2-C-Methyl-D-erythritol 4-phosphate (MEP), 4-(Cytidine 5' -diphospho)-2-C-methyl-D-erythritol (CDPME), 4-(Cytidine 5'-diphospho)-2-C-methyl-D-erythritol (DEP-ME-2P), 2-C-Methyl-D-erythritol 2,4-cyclodiphosphate (MEcPP), 1-Hydroxy-2-methyl-2-butenyl 4-diphosphate (HMBPP).

\subsection{Vitamin K2 (Menaquinone) and Peptidoglycan Biosynthesis Pathways in Mouse NAFLD-Phenotypes and Gut Microbiomes of Cirrhosis Patients}

In polyprenol diphosphate biosynthesis, IPP is recursively added to geranyl diphosphate (GPP) or farnesyl diphosphate (FPP) for polyprenol biosynthesis used in vitamin K2 (a.k.a., menaquinone) and peptidoglycan biosynthesis [41,42]. TCDD enriched for heptaprenyl diphosphate synthase (EC 2.5.1.30) with major contributions from L. reuteri and L. johnsonii (Figure 5). 

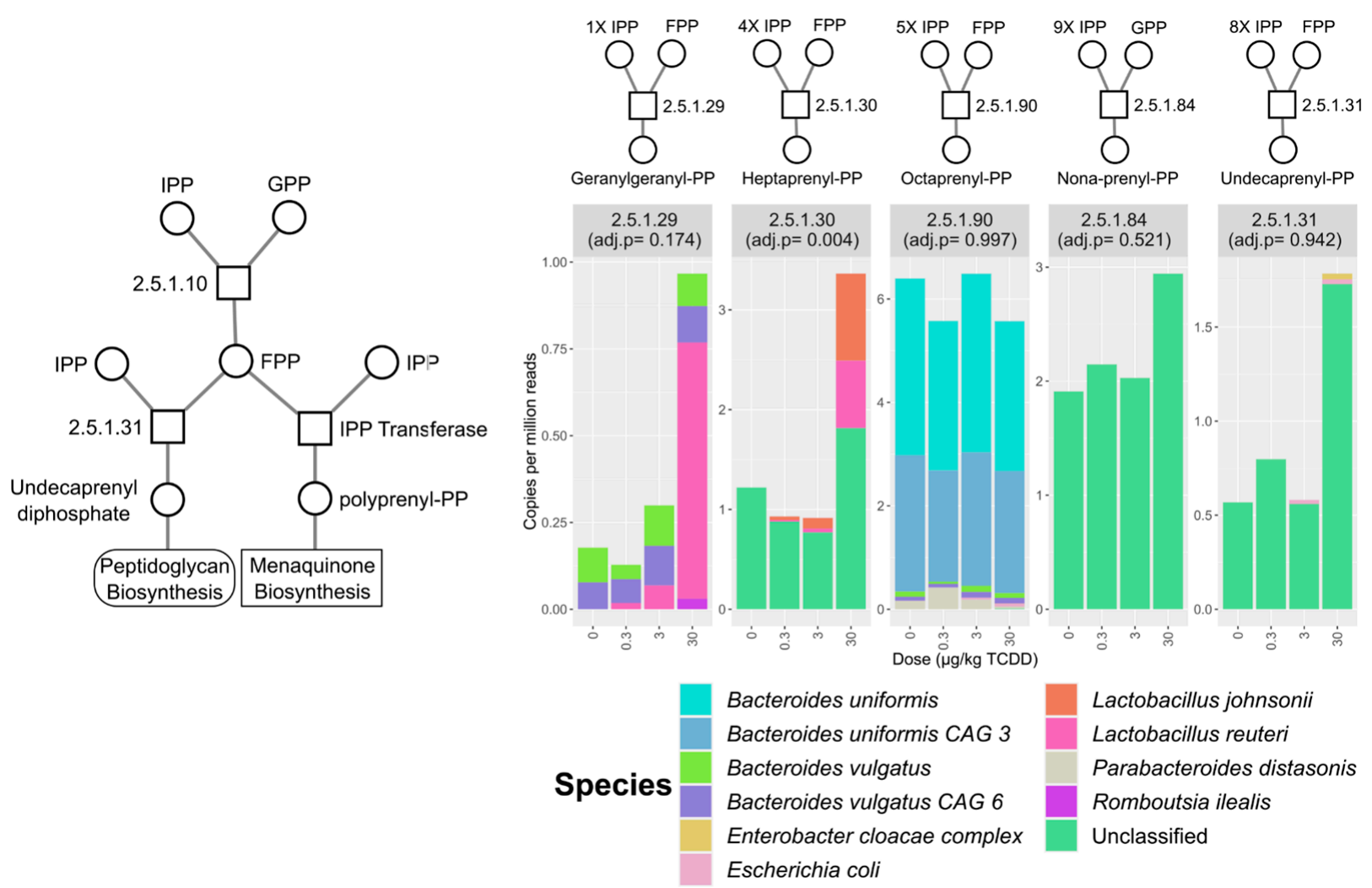

Figure 5. Relative abundance of polyprenol transferase EC annotations identified in the mouse cecum metagenomic dataset. Stacked bar plots represent mean relative abundance of grouped EC numbers $(n=3)$ and represent identified species that contributed to mean total abundance for each treatment group. The number of isopentenyl diphosphate (IPP) and farnesyl diphosphate (FPP) molecules used for respective polyprenol biosynthesis are also denoted. Adjusted $p$-values were determined by the Maaslin2 R package. Abbreviations: isopentenyl diphosphate (IPP), geranyl diphosphate (GPP), polyprenyl diphosphate (polyprenyl-PP).

As bacterial cell wall restructuring has been reported in response to bile acids and different levels of isoprenoid biosynthesis pathways were identified, peptidoglycan biosynthesis was also assessed [43]. Most genes encoding enzymes required for peptidoglycan biosynthesis were present in the metagenomic dataset (Figure 6A) with no changes observed following TCDD treatment.

However, serine-type D-Ala-D-Ala carboxypeptidase (Figure 6a, EC 3.4.16.4, step 14), responsible for peptidoglycan polymer crosslinking, trended upwards. Additionally, most peptidoglycan biosynthesis EC numbers had annotations to L. reuteri (Figure 6B). Overall, TCDD did not alter peptidoglycan synthesis related gene levels.

De novo menaquinone biosynthesis requires chorismate and the addition of a polyprenol diphosphate (i.e., geranyl-geranyl diphosphate) (Figure 7A). Two alternative pathways exist for menaquinone biosynthesis, the o-succinylbenzoate or futalosine route [44]. Only a few EC number annotations were detected for the futalosine pathway (EC 4.2.1.151 and EC 2.5.1.120), while all EC numbers were identified for the complete o-succinylbenzoate menaquinone pathway (Figure 7A). 
A

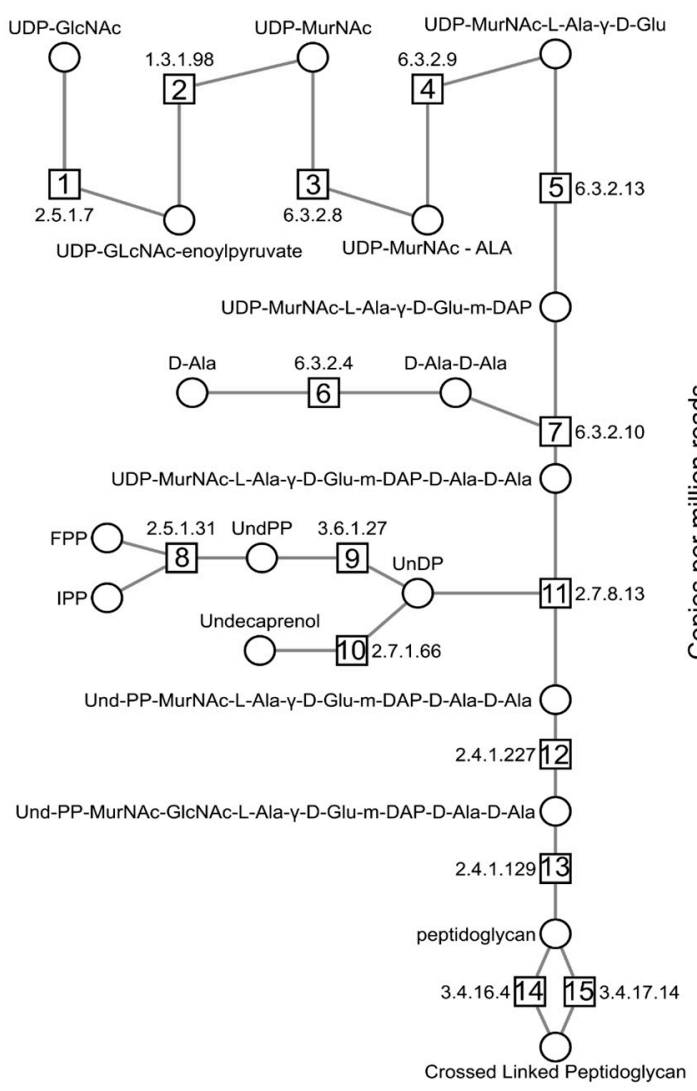

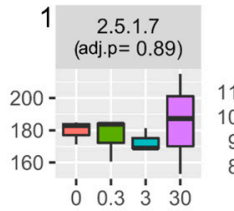
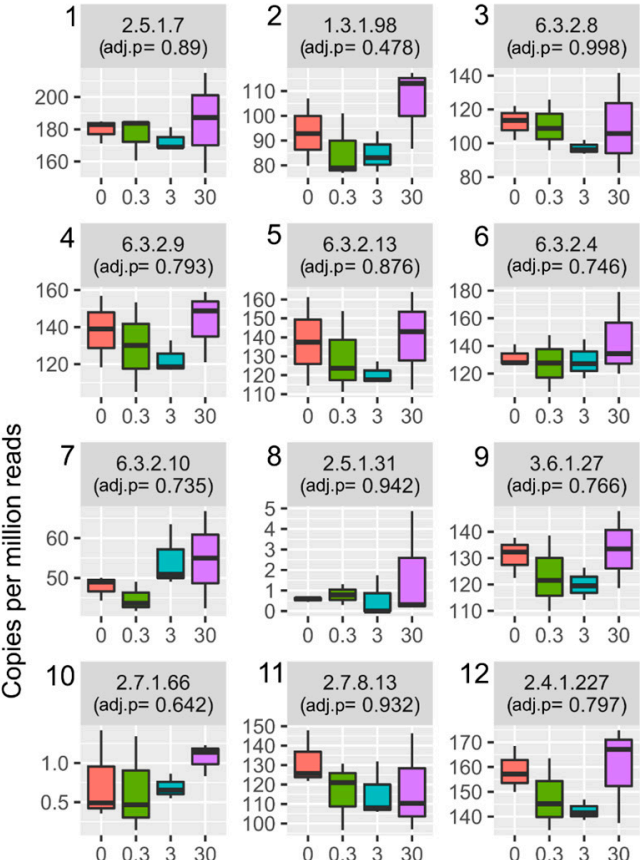

$\begin{array}{lllll}0 & 0.3 & 3 & 30\end{array}$
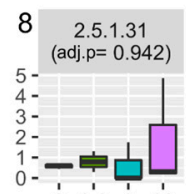

$6 \quad 6.324$

$6 \begin{gathered}6.3 .2 .4 \\ (a d j . p=0.746)\end{gathered}$

$80-$

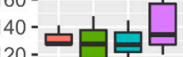

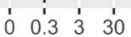

$9 \quad 3.6 .1 .27$

(adj.p=0.766)

130-官口吕

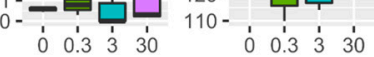

$11 \begin{gathered}2.7 .8 .13 \\ \text { (adj.p }=0.932)\end{gathered}$

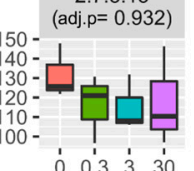

$12 \quad 2.4 .1 .227$

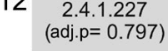

$170-$

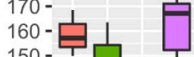

$(50-$

$\begin{array}{llll}1 & 1 & 1 & 1 \\ 0 & 0.3 & 3 & 30\end{array}$

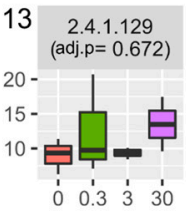

$14 \begin{gathered}3.4 .16 .4 \\ (\text { adj.p }=0.324)\end{gathered}$

14 -

12- 1.5 -

- $\square$ 店

- -

$\begin{array}{llll}1 & 0.3 & 3 & 30\end{array}$

Dose $(\mu \mathrm{g} / \mathrm{kg}$ TCDD)
$15 \quad 3.4 .17 .14$

$1.5-\quad$

$1.0-$

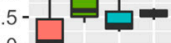

$0-\frac{\square}{1} \quad \begin{array}{lll}1 & 1 & 1\end{array}$

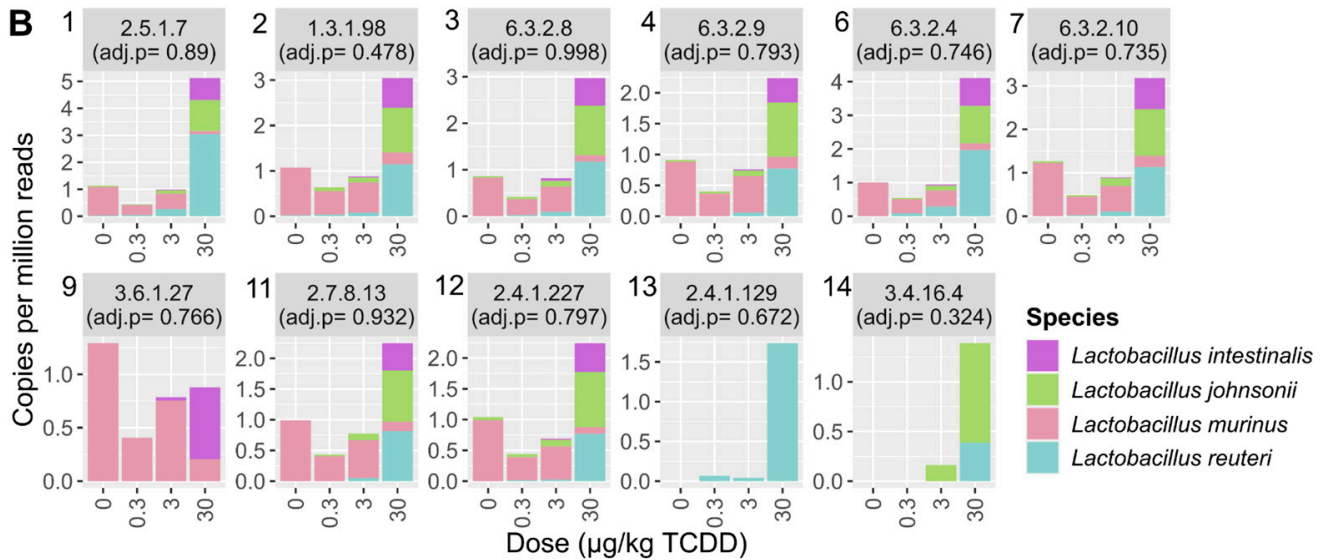

Figure 6. Peptidoglycan biosynthesis was unchanged by TCDD. (A) Relative abundance of peptidoglycan biosynthesis EC numbers identified in the metagenomic dataset. (B) Relative abundance of only Lactobacillus species classified to peptidoglycan biosynthesis EC numbers. Individual boxplots are numbered with the EC number matching the enzymatic step in pathway schematic. Adjusted $p$-values (adj. $p$ ) were determined by MAASLIN2. Abbreviations: UDP-Nacetyl-alpha-D-glucosamine (UDP-GlcNac), UDP-N-acetylmuramate (UDP-MurNAc), UDP-N-acetyl-alpha-D-muramoyl-Lalanine (UDP-MurNAc-ALA), UDP-N-acetyl-alpha-D-muramoyl-L-alanyl-D-glutamate(UDP-MurNAc-Ala-D-Glu), UDP$\mathrm{N}$-acetylmuramoyl-L-alanyl-gamma-D-glutamyl-meso-2,6-diaminopimelate (UDP-MurNAc-Ala-D-Glu-m-DAP), D-AlanylD-alanine (D-Ala-D-Ala), UDP-N-acetylmuramoyl-L-alanyl-D-glutamyl-6-carboxy-L-lysyl-D-alanyl-D-alanine (UDPMurNAc-Ala-D-Glu-m-DAP-D-Ala-D-Ala), Undecaprenyl-diphospho-N-acetylmuramoyl-L-alanyl-D-glutamyl-meso-2,6diaminopimeloyl-D-alanyl-D-alanine (Und-PP-MurNAc-Ala-D-Glu-m-DAP-D-Ala-D-Ala),Undecaprenyl-diphospho-Nacetylmuramoyl-(N-acetylglucosamine)-L-alanyl-D-glutamyl-meso-2,6-diaminopimeloyl-D-alanyl-D-alanine (Und-PPMurNAc-GlcNAc-Ala-D-Glu-m-DAP-D-Ala-D-Ala). 

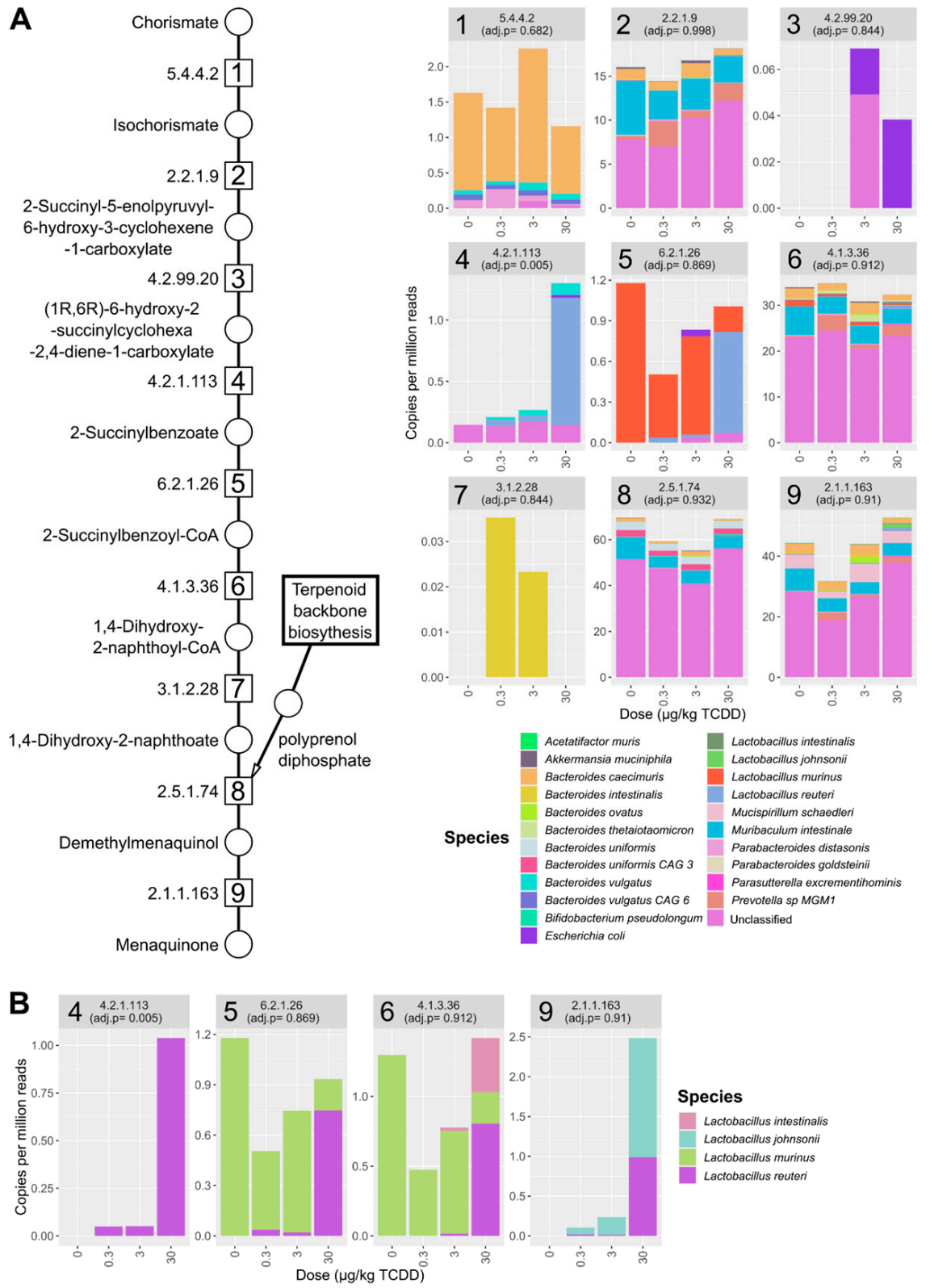

Figure 7. TCDD-elicited effects on menaquinone biosynthesis. (A) Relative abundance of menaquinone biosynthesis EC annotations identified in the metagenomic dataset. Individual stacked bar plots are labeled with the EC number matching the enzymatic step in pathway schematic. Stacked bar plots of annotated EC numbers involved in menaquinone biosynthesis. Values are mean relative abundance $(n=3)$ classified to the respective species and in cecum samples from male C57BL/ 6 mice following oral gavage with sesame oil vehicle or $0.3,3$, or $30 \mu \mathrm{g} / \mathrm{kg}$ TCDD every 4 days for 28 days. (B) Menaquinone biosynthesis EC numbers classified to Lactobacillus species in the cecum metagenomic datasets. Adjusted $p$-values (adj. $p$ ) were determined by Maaslin2 R package.

In the mouse cecum dataset, species contributing to o-succinylbenzoate menaquinone biosynthesis pathway included Escherichia coli, several Bacteroides (e.g., Bacteroides vulgatus and Bacteroides caecimuris), and Lactobacillus species (e.g., L. reuteri) (Figure 7B and Table S6). No one species was annotated to the entire set of enzymes needed for de novo biosynthesis from chorismate, however $B$. vulgatus was annotated for 6 out of 9 genes in the pathway (Table S6). O-Succinylbenzoate synthase (Figure 6A, EC 4.2.1.113, step 4) was increased by $30 \mu \mathrm{g} / \mathrm{kg}$ TCDD with L. reuteri being the major contributor to relative abundance (Figure 7 , step 4$)$. 
Lactobacillus species annotated to menaquinone biosynthesis included $L$. reuteri, $L$. murinus, and L. johnsonii. Among annotated menaquinone biosynthesis EC numbers, $L$. reuteri was among the identified Lactobacillus species that had the highest relative abundance and most menaquinone EC annotations (Figure 7B). L. reuteri also had annotations in samples for EC numbers involved in the final steps of the shikimate pathway responsible for chorismate biosynthesis (Table S7).

In the cirrhosis samples, several EC numbers representing the initial menaquinone biosynthesis steps were also increased in compensated and decompensated patients (Figure 8, steps 1,35), including o-succinylbenzoate synthase (Figure 8, EC 4.2.1.113, step 4).
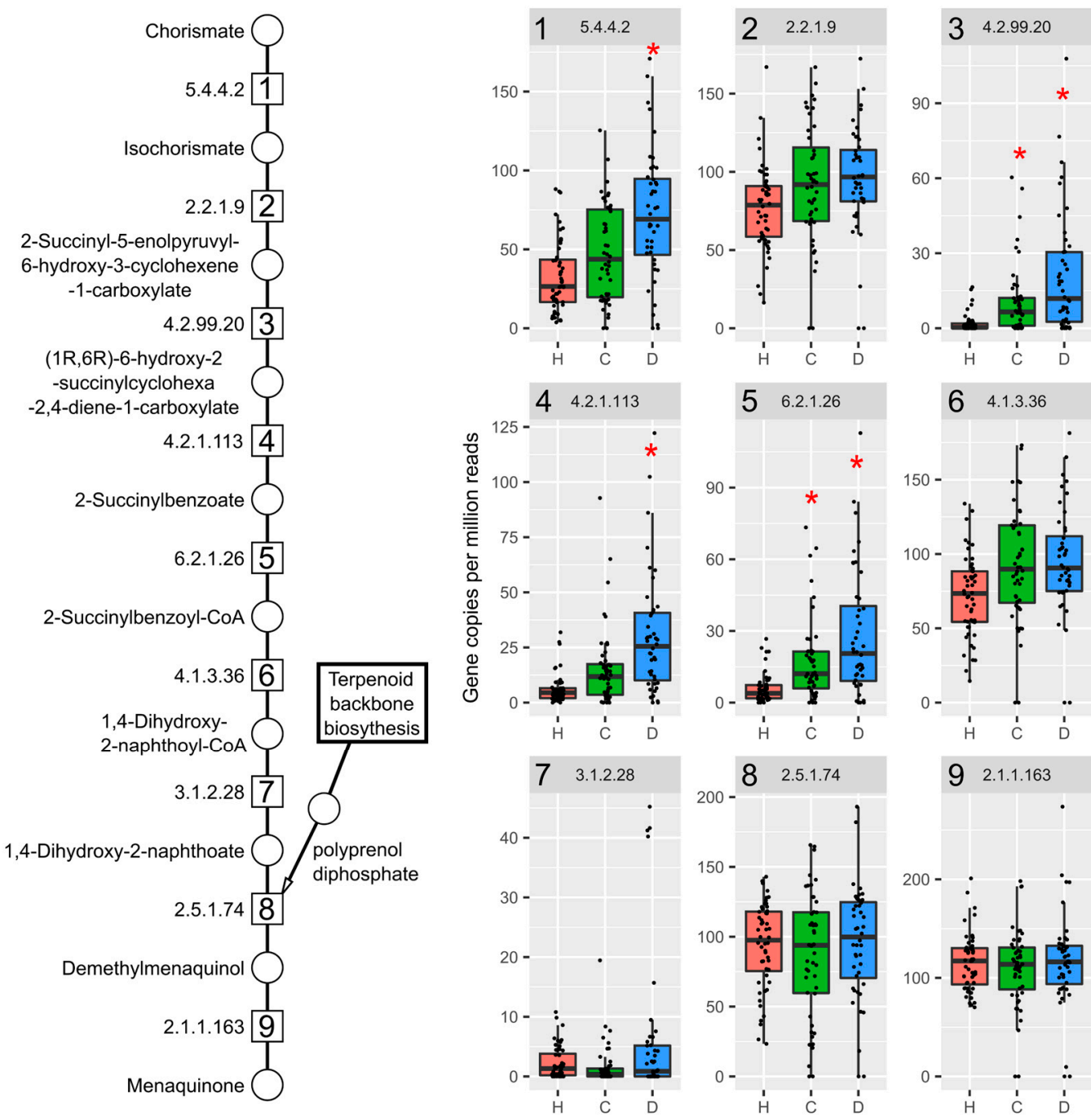

Figure 8. Menaquinone biosynthesis genes are increased in cirrhotic patients. Humann3 analysis of fecal metagenomic dataset of patients with healthy $(\mathrm{H}$, red, $n=52)$, compensated ( , green, $n=48)$, or decompensated ( $\mathrm{D}$, blue, $n=44)$ liver cirrhosis diagnosis for EC numbers in menaquinone biosynthesis. Individual box plots are labeled with the EC number matching the enzymatic step in pathway schematic. Significance is denoted with a red asterisk (*; adjusted $p$-values $<0.05)$ with the healthy group as reference.

However, L. reuteri was not among species classified to this EC number. Species classified to all EC numbers comprising the complete pathway included E. coli, and Klebsiella species, such as K. pneumoniae and Citrobacter species. L. reuteri, were not annotated to any menaquinone biosynthesis genes in healthy or compensated patients, but several EC numbers in the decompensated group (EC 6.2.1.26, 4.1.3.6, and 2.1.1.163), which are involved in later stages of menaquinone biosynthesis (Table S8). 


\section{Discussion}

Previous studies have reported that TCDD elicited NAFLD-like pathologies, dysregulated bile acid metabolism and gut microbiome dysbiosis $[9,11,12,28,30]$. This study further elucidated the shifts in the gut microbiota associated with TCDD treatment using shotgun metagenomic sequencing. We show that TCDD dose-dependently shifted the gut microbiota composition by enriching for Lactobacillus species, consistent with hepatic disruption of host and microbial bile acid metabolism. In addition, TCDD enriched for genes involved in mevalonate dependent isoprenoid precursor biosynthesis and menaquinone biosynthesis, crucial for microbial cell growth and survival. Over-representation of these microbial associated pathways were also identified in human cirrhosis stool metagenomics datasets.

TCDD-elicited gut dysbiosis is in agreement with observed effects in published in vivo studies following treatment with endogenous (i.e., FICZ) and exogenous (i.e., TCDD and TCDF) AhR agonists $[8,9,11,28-30]$. More specifically, we observed an increased Firmicutes/Bacteroides ratio with dose-dependent increases in Lactobacillus species [28,30]. Lactobacillus species are often associated with NAFLD and with increased abundances in patients with diabetes and liver fibrosis [45].

Probiotic Lactobacillus species, including L. reuteri supplementation, have also been reported to alleviate NAFLD pathologies by reducing steatosis [46], fibrosis [47], insulin resistance [48] and serum cholesterol levels [49]. However, Lactobacillus species supplementation may also exacerbate fibrosis [50]. In humans and mice, L. reuteri supplementation can modulate the gut microbiota and alter bile acid metabolism. L. reuteri enrichment also approached comparable levels compared to samples from humans and mice administered probiotic supplementation [51,52].

We observed a species-specific increase of $L$. reuteri with a concurrent decrease in $L$. murinus suggesting shifts in Lactobacillus composition at the species and/or strain levels. Further, decreased abundance of L. murinus has been reported in human NAFLD [53]. Other taxa enriched following treatment included Turicibacter sanguinis, an anaerobic grampositive bacillus commonly found in animals, including humans [54]. Interestingly, $T$. sanguinis has been shown to deconjugate bile acids and metabolize serotonin affecting lipid and steroid metabolism [54,55].

Quantitative trait locus analysis correlated T. sanguinis abundance with cholic acid levels and expression of the intestinal bile acid transporter Slc10a2 [54]. Both cholic acid levels and Slc10a2 expression are dose-dependently increased by TCDD [9]. Consequently, the dose-dependent taxonomic shift in Lactobacillus and Turicibacter species known to deconjugate conjugated bile acids is consistent with increased levels of secondary bile acids following TCDD treatment.

Some host relevant intestinal health and homeostatic effects can be attributed to Lactobacillus species mediated by bile salt hydrolases (BSHs), which are responsible for deconjugation reactions, the gateway step for conversion of conjugated primary bile acid to secondary bile acids [56]. A majority of Lactobacillus species possess BSHs, often containing multiple different gene copies within their genome, some with different bile acid substrate preferences $[33,38]$.

However, the presence of $b s h$ sequences does not simply infer bile acid tolerance as growth inhibition and reduced fitness is also possible depending on the conjugated or deconjugated bile acids present and/or BSH specificity $[33,38,57]$. For example, L. gasseri bsh knockout mutants exhibit increased fitness compared to wild type strains [38]. Interestingly, L. gasseri $b s h$ sequences were not identified despite increased L. gasseri abundance following TCDD treatment. Our $b s h$ analysis also found TCDD enriched Lactobacillus-associated sequences that may impart bile acid tolerance.

For example, the $b s h$ sequence enriched by TCDD annotated to L. johnsonii (RefSeq ID: EGP12391) (Table S3) exhibited higher substrate specificity for glycine over taurine conjugated bile acids [58]. In a companion study using the dose response and treatment regimen, Fader et al. reported TCDD increased serum DCA levels $\sim 80$ fold, with only a $\sim$ two-fold increase in serum GDCA levels [9]. In contrast, hepatic taurolithocholic acid 
(TLCA) levels were increased 233 fold while serum lithocholic acid increased only four fold following TCDD treatment.

Moreover, glycine conjugated bile acids, including GDCA, are more toxic towards Lactobacillus species than taurine conjugated bile acids $[33,59,60]$. Increased levels of BSH with a substrate preference for glycine conjugated bile acid may partially explain select Lactobacillus species enrichment. Further, both TLCA and DCA are potent FXR and GPBAR1 agonists that regulate the lipid, glucose, and bile acid metabolism [61,62]. Consequently, shifts in microbial secondary bile acids by Lactobacillus species may play a role in TCDD elicited gut dysbiosis impacting host regulation of energy homeostasis.

Coincident with increased levels of $b s h$ was the dose-dependent increase in genes from the mevalonate-dependent isoprenoid biosynthesis, the pathway also used in mammals for cholesterol biosynthesis. The MEP pathway is the predominant isoprenoid biosynthesis pathway among gut microbiota while the mevalonate-dependent pathway is only found in select bacteria, including Lactobacillus and Streptococcus species [63]. The output from either pathway is farnesyl diphosphate (FPP) and geranyl diphosphate (GPP), substrates required for polyprenol biosynthesis used in menaquinone and cell wall biosynthesis.

Menaquinones are utilized by bacteria for anaerobic/aerobic respiration, providing antioxidant activity with menaquinone supplementation affecting the gut microbiome [64]. In the context of L. reuteri, we observed genes annotated to the shikimate pathway, which is responsible for chorismate biosynthesis, a precursor for aromatic amino acids and the naphthoquinone head group of menaquinone, as well as genes involved in de novo menaquinone biosynthesis. While the complete biosynthesis pathway was not present in L. reuteri, it is consistent with other metagenomic reports of incomplete menaquinone biosynthesis pathways in gut Lactobacillus species [44].

It has been proposed that Lactobacillus species may participate in later menaquinone biosynthesis steps through the uptake of intermediates, such as o-succinylbenzoate from other bacteria or dietary sources [44]. In addition, the ability to utilize menaquinones for respiration is typically not associated with Lactobacillus species. However, some lactic acid bacteria, including $L$. reuteri strains, demonstrate the ability to respire when menaquinone and heme are supplemented $[65,66]$.

Metagenomic analysis also identified the mevalonate-dependent pathway enrichment in fecal samples from patients with cirrhosis. The mevalonate-dependent pathway is reported to be increased in fibrosis patients with autoimmune pathologies [67]. Isoprenoid biosynthesis pathways are also elevated in the lung microbiome of cystic fibrosis patients, with the MEP pathway enriched rather than the mevalonate route [68]. The association between fibrosis and isoprenoid biosynthesis enrichment warrants further investigation in the context of potential mechanisms contributing to bacterial fitness and/or fibrosis.

Increased abundance of the mevalonate-dependent biosynthesis pathway could also be a biomarker of Lactobacillus and Streptococcus proliferation that is often associated with non-alcoholic steatohepatitis (NASH)/fibrosis [21,45]. We identified enrichment of the mevalonate-dependent pathway in both mouse and human microbiomes, whereas the complete pathway was primarily annotated to Streptococcus and Lactobacillus species (Table S7).

Other factors, such as simvastatin and proton pump inhibitors (PPI), that are commonly prescribed for NAFLD patients may also impact these microbial pathways. Simvastatin, which is primarily excreted in the feces [69], has been reported to reduce bacterial growth by directly inhibiting bacterial HMG-CoA synthesis while PPIs inhibit Streptococcus species growth [70-72]. These microbiome-drug interactions highlight off target effects that should be considered when investigating novel NAFLD treatments, such as new drug development and/or probiotic interventions.

In addition to increased mevalonate-dependent isoprenoid biosynthesis genes in cirrhotic patients, menaquinone biosynthesis gene abundance was also increased. This suggests taxa with the ability to produce menaquinone may have a competitive advantage when intestinal environmental conditions shift during disease progression. In cirrhosis 
patients, E. coli and B. vulgatus were associated with genes providing a majority of the menaquinone biosynthesis capacity. These species are also increased in human NAFLD [73].

Similar to the results in mice exposed to TCDD, L. reuteri was associated with several menaquinone biosynthesis genes and only detected in decompensated cirrhosis patients but lacked the complete pathway (Table S10). In cirrhosis patients, it is unclear whether L. reuteri is participating in menaquinone metabolism and/or benefiting from increased abundance of species, like $E$. coli that are capable of producing menaquinones.

This study was designed to account for factors affecting gut microbiota analysis, including cage effects, coprophagia, and circadian rhythms [74]. Significant shifts in taxa were observed in Lactobacillus species. However, the small group size $(n=3)$ following adjustment for multiple testing lacked sufficient power to confirm more subtle shifts, such as the two fold enrichment of Lachnospiraceae A4, an abundant community member associated with bsh sequences.

Samples were also collected within the same Zeitgeber period to account for possible variations in relative microbiota levels due to circadian rhythm/diurnal regulation. In fact, L. reuteri is one gut microbiome member demonstrating changes in relative abundance in human samples due to circadian/diurnal regulation [75]. TCDD disrupted diurnal regulation of hepatic gene expression, including bile acid biosynthesis genes, which may contribute to $L$. reuteri enrichment [76].

The same dose range (sesame oil vehicle and 0.01-30 $\mu \mathrm{g} / \mathrm{kg}$ TCDD) and dosing regimen (every 4 days for 28 days) has been used in previous studies to examine TCDDelicited effects in mice relevant to NAFL D development and progression [9,11-15,30,76-78]. This model recapitulates similar NAFLD progression and pathologies with hepatocyte vacuolization at doses as low as $0.3 \mu \mathrm{g} / \mathrm{kg}$ TCDD. In addition, immune cell infiltration (steatohepatitis) was observed at doses as low $3 \mu \mathrm{g} / \mathrm{kg}$ TCDD with collagen deposition (fibrosis) reported at $30 \mu \mathrm{g} / \mathrm{kg}$ TCDD [9].

Using this study design, TCDD also disrupted the bile acid metabolism and enterohepatic circulation with increased hepatic and serum total bile acids and secondary bile acid DCA [9]. Likewise, increased serum bile acid levels, including DCA, have been reported in patients with steatohepatitis and fibrosis [79-82]. Furthermore, NAFLD patients with increased bile acid levels have increased levels of bacterial genes from the bai operon associated with $7 \alpha$-dehydroxylation of bile acids leading to the production of DCA from cholic acid [83].

Although the consequences of TCDD-elicited immune system effects on the gut microbiome were not assessed in this study, it is most likely a factor impacting $L$. reuteri enrichment. TCDD causes macrophage and dendritic cell migration out of the lamina propria with increased accumulation in the liver, possibly exacerbating hepatic inflammation and affecting intestinal immune responses [14]. The ability of L. reuteri to produce AhR ligands, upregulate IL-22, and associate with the mucosa and Peyer's patches provides geographical proximity for immune/microbiome crosstalk mediated by the AhR $[26,84,85]$.

In addition to immune cell regulation, TCDD increased bone formation and decreased bone marrow adiposity [86]. Interestingly, L. reuteri supplementation also increased bone density, but only when mice were induced towards an inflammatory state [87]. Overall, the dose-dependent increase in L. reuteri levels is consistent with increased bile acid levels, disruption of circadian/diurnal regulation and increased bone density $[9,75,86,87]$.

In summary, Lactobacillus species were dose-dependently increased following AhR activation by TCDD concurrent with the increase in bsh genes and increased primary and secondary bile acids. Specifically, L. reuteri, a keystone gut microbiome species is involved in the microbial metabolism of bile acids and AhR ligands. The large and uniform enrichment of $L$. reuteri in this study also suggests environmental pressures, such as increased levels of bile acids and antimicrobial peptides elicited by AhR activation, may provide a complementary niche for $L$. reuteri that possess a gene repertoire not found in the closely related L. murinus. 
We also provide evidence regarding how the L. reuteri metabolism could impact the AhR, FXR, and GPBAR1 signaling pathways, placing L. reuteri at the crossroads of bacterial/host interactions affecting glucose, bile acid, and immune regulation. Whether these microbial shifts in metabolism are adaptive and limit the intensity of adverse consequences or exacerbate steatosis to steatohepatitis with fibrosis progression warrants further investigation.

\section{Materials and Methods}

\subsection{Animal Treatment}

Postnatal day 25 (PND25) male C57BL/ 6 mice weighing within 10\% of each other were obtained from Charles River Laboratories (Kingston, NY, USA) and housed and treated as previously described [9]. Briefly, mice were housed in Innovive Innocages (San Diego, CA, USA) containing ALPHA-dri bedding (Shepherd Specialty Papers, Chicago, IL, USA) in a $23{ }^{\circ} \mathrm{C}$ environment with $30-40 \%$ humidity and a $12 \mathrm{~h} / 12 \mathrm{~h}$ light/dark cycle. Aquavive water (Innovive) and Harlan Teklad 22/5 Rodent Diet 8940 (Madison, WI, USA) were provided ad libitum.

The rodent diet is a fixed formula complete diet with an energy density of $3.0 \mathrm{kcal} / \mathrm{g}$ and a nutrient ingredient composition, including $22 \%$ protein, $5.5 \%$ fat, and $40.6 \%$ carbohydrate. Mice (PND29) were orally gavaged at the beginning of the light cycle (between Zeitgeber time 0-3) with $0.1 \mathrm{~mL}$ sesame oil vehicle (Sigma-Aldrich, St. Louis, MO, USA) or $0.3,3$ and $30 \mu \mathrm{g} / \mathrm{kg}$ body weight TCDD (AccuStandard, New Haven, CT, USA) every 4 days for 7 total exposures ( $n=3$ per treatment group). The study was conducted in three cohorts with mice housed separately among treatment groups for a total of 9 mice per treatment group.

In each cohort, three mice were housed per treatment group, and one mouse was randomly selected from each treatment group per cohort $(n=3$ per treatment group for the metagenomic analysis) to account for coprophagia and ensure reproducibility. The first gavage was administered on day 0 of the study. On day 28, vehicle- and TCDD-treated mice (fasted for $6 \mathrm{~h}$ with access to water) were weighed and euthanized by $\mathrm{CO}_{2}$ inhalation between Zeitgeber time 0-3. Upon collection, cecums were immediately flash frozen in liquid nitrogen and stored at $-80^{\circ} \mathrm{C}$ until analysis. All animal handling procedures were performed with the approval of the Michigan State University (MSU) Institutional Animal Care and Use Committee.

\subsection{Metagenomic Sequencing}

Microbial DNA from cecum contents ( $25 \mathrm{mg}$ ) was extracted using the FastDNA spin kit for soil (SKU 116560200, MP Biomedicals, Santa Ana, CA, USA). Extracted DNA was submitted to Novogene (Sacramento, CA, USA) for quality control, library preparation, and 150-bp paired-end sequencing at a depth 136-157 million reads using an Illumina NovaSeq 6000. Reads aligning to the C57BL/6 Mus musculus genome (https:/ / www.ncbi.nlm.nih. gov / assembly/GCF_000001635.26/, NCBI genome assembly: GRCm38.p6, accessed on 10 October 2020) were identified, flagged, and removed using bowtie2 [88], SamTools [89] and bedtools [90]. For human metagenomic analysis, reads were filtered against the human genome (https: / / www.ncbi.nlm.nih.gov/assembly/GCF_000001405.13/, NCBI genome assembly: GRCh37/hg19, accessed on 10 January 2021) using the Kneaddata bioinformatics tool developed at the Huttenhower Lab (https:/ / github.com/biobakery/ kneaddata, accessed on 10 January 2021).

\subsection{Metagenomic Taxonomic Analysis}

Kaiju was used for taxonomic analysis of mouse cecum metagenomic dataset. The reference database used was the progenomes database downloaded from the kaiju webserver (https:/ / kaiju.binf.ku.dk/database/kaiju_db_progenomes_2020-05-25.tgz, accessed on 15 October 2020). Multivariate association between dose and taxonomy relative abundances used Maaslin2 (https:/ / github.com/biobakery/Maaslin2, accessed on 15 October 
2020) [91] with the following default settings used: normalization (total sum scaling), analysis method (general linear model), and Benjamini-Hochberg multiple test correction. Adjusted $p$-values for Maaslin2 analysis used dose (sesame oil vehicle (0), 0.3, 3, or $30 \mu \mathrm{g} / \mathrm{kg}$ TCDD) as the fixed effect, which was treated as continuous variable and the vehicle set for reference. For comparison of taxonomy between vehicle and $30 \mu \mathrm{g} / \mathrm{kg}$ TCDD treatment groups, DeSeq 2 was used to determine adjusted $p$-values using default settings [92].

\subsection{Metagenomic Functional Analysis}

The HUMAnN 3.0 bioinformatic pipeline [93] was used with default settings to classify reads to UniRef90 protein identifications using UniProt's UniRef90 protein data base (January 2019, accessed on 15 January 2021). Reads aligned to UniRef90 identifications were mapped to enzyme commission (EC) number entries using the human_regroup_table tool. Read abundance was normalized to gene copies per million reads (CPM) using the human_renorm_table tool. Multivariate association between dose and enzyme commission number relative abundance used Maaslin2 with same settings used for taxonomy analysis.

Xander (a gene-targeted assembler, https:/ / github.com/rdpstaff/Xander_assembler, accessed on 5 February 2021) was used to annotate and quantify bile salt hydrolase sequences with the following settings: $\mathrm{k}$-mer size $=45$, filter size $=35$, minimum assembled contig bit score $=50$, and minimum assembled protein contigs $=100$ [94]. Reference DNA and protein $b s h$ sequences used for Xander were downloaded from FunGenes Gene Repository and are listed in supplementary material (Tables S9 and S10) [95]. For RefSeq bsh sequence analysis, relative abundance was determined by normalizing to total abundance of $r p l B$ sequences also determined by Xander per sample. Significance was determined with Maaslin2 with the same settings used for taxonomy analysis.

Human metagenomic data from the National Center for Biotechnology Information (NCBI) Sequence Read Archive (SRA) under accession number PRJEB6337 (https: / / www.ncbi.nlm.nih.gov/bioproject/PRJEB6337/, accessed on 25 March 2021) was analyzed using the same HUMAnN 3.0 pipeline as cecum metagenomic data. Fecal shotgun metagenomic samples from Chinese patients were defined as healthy $(n=52)$ or cirrhotic with subclassifications of compensated $(n=48)$ or decompensated $(n=44)$ by the authors [39].

Cirrhosis was diagnosed by either biopsy, clinical evidence of decompensation, or other metrics, including radiological evidence of liver nodularity and intra-abdominal varices in a patient with chronic liver disease [39]. The subclassification was used as fixed effect for analysis with healthy as the reference category. Again, Maaslin2 was used with settings used for mouse functional analysis with diagnosis as a fixed effect with healthy diagnosis as reference to determine adjusted $p$-values for compensated and decompensated patient designations.

Supplementary Materials: The following are available online at https:/ /www.mdpi.com/article/10 .3390/ijms222212431/s1.

Author Contributions: Conceptualization, R.R.F. and T.R.Z.; methodology, R.R.F., T.R.Z.; writingoriginal draft preparation, R.R.F.; writing-review and editing, R.R.F., T.R.Z.; funding acquisition, T.R.Z.; formal analysis, R.R.F.; visualization, R.R.F; data curation, R.R.F.; Methodology R.R.F., T.R.Z.; validation, R.R.F.; project administration, T.R.Z.; investigation, R.R.F., T.R.Z.; All authors have read and agreed to the published version of the manuscript.

Funding: This work was funded by the NIEHS Superfund Research Program (NIEHS SRP P42ES004911), and NIEHS R01ES029541 to T.R.Z. T.R.Z. is partially supported by AgBioResearch at Michigan State University (MICL02603). R.R.F. is supported by the NIEHS Multidisciplinary Training in Environmental Toxicology (NIEHS EHS T32ES007255).

Institutional Review Board Statement: All animal handling procedures were performed with the approval of the Michigan State University (MSU) Institutional Animal Care and Use Committee, in accordance with ethical guidelines and regulations under IACUC ID: PROTO201800043. 
Informed Consent Statement: Informed consent was obtained from all subjects involved in the human metagenomics study [39].

Data Availability Statement: Quality filtered mouse metagenomic data from this study can be found at the NCBI Sequence Read Archive (SRA) (https: / /www.ncbi.nlm.nih.gov/ bioproject/ PRJNA719224/, accessed on 10 August 2021) under the accession ID PRJNA719224. The human fecal metagenomic data presented in this study is openly available and can be found at the NCBI SRA under the accession number PRJEB6337 (https:/ / www.ncbi.nlm.nih.gov/bioproject/PRJEB6337/, accessed on 25 March 2021) [39].

Conflicts of Interest: The authors declare no conflict of interest. The funders had no role in the design of the study; in the collection, analyses, or interpretation of data; in the writing of the manuscript, or in the decision to publish the results.

\section{References}

1. Younossi, Z.M.; Koenig, A.B.; Abdelatif, D.; Fazel, Y.; Henry, L.; Wymer, M. Global Epidemiology of Nonalcoholic Fatty Liver Disease-Meta-Analytic Assessment of Prevalence, Incidence, and Outcomes. Hepatology 2016, 64, 73-84. [CrossRef]

2. Anstee, Q.M.; Targher, G.; Day, C.P. Progression of NAFLD to Diabetes Mellitus, Cardiovascular Disease or Cirrhosis. Nat. Rev. Gastroenterol. Hepatol. 2013, 10, 330-344. [CrossRef] [PubMed]

3. Tilg, H.; Moschen, A.R. Evolution of Inflammation in Nonalcoholic Fatty Liver Disease: The Multiple Parallel Hits Hypothesis. Hepatology 2010, 52, 1836-1846. [CrossRef] [PubMed]

4. Cave, M.; Appana, S.; Patel, M.; Falkner, K.C.; McClain, C.J.; Brock, G. Polychlorinated Biphenyls, Lead, and Mercury Are Associated with Liver Disease in American Adults: NHANES 2003-2004. Environ. Health Perspect. 2010, 118, $1735-1742$. [CrossRef] [PubMed]

5. Zheng, S.; Yang, Y.; Wen, C.; Liu, W.; Cao, L.; Feng, X.; Chen, J.; Wang, H.; Tang, Y.; Tian, L.; et al. Effects of Environmental Contaminants in Water Resources on Nonalcoholic Fatty Liver Disease. Environ. Int. 2021, 154, 106555. [CrossRef] [PubMed]

6. Kumar, J.; Lind, L.; Salihovic, S.; van Bavel, B.; Ingelsson, E.; Lind, P.M. Persistent Organic Pollutants and Liver Dysfunction Biomarkers in a Population-Based Human Sample of Men and Women. Environ. Res. 2014, 134, 251-256. [CrossRef] [PubMed]

7. Rantakokko, P.; Männistö, V.; Airaksinen, R.; Koponen, J.; Viluksela, M.; Kiviranta, H.; Pihlajamäki, J. Persistent Organic Pollutants and Non-Alcoholic Fatty Liver Disease in Morbidly Obese Patients: A Cohort Study. Environ. Health 2015, 14, 79. [CrossRef]

8. Zhang, L.; Nichols, R.G.; Correll, J.; Murray, I.A.; Tanaka, N.; Smith, P.B.; Hubbard, T.D.; Sebastian, A.; Albert, I.; Hatzakis, E.; et al. Persistent Organic Pollutants Modify Gut Microbiota-Host Metabolic Homeostasis in Mice through Aryl Hydrocarbon Receptor Activation. Environ. Health Perspect. 2015, 123, 679-688. [CrossRef] [PubMed]

9. Fader, K.A.; Nault, R.; Zhang, C.; Kumagai, K.; Harkema, J.R.; Zacharewski, T.R. 2,3,7,8-Tetrachlorodibenzo- $p$-Dioxin (TCDD)Elicited Effects on Bile Acid Homeostasis: Alterations in Biosynthesis, Enterohepatic Circulation, and Microbial Metabolism. Sci. Rep. 2017, 7, 5921. [CrossRef]

10. Petriello, M.C.; Hoffman, J.B.; Vsevolozhskaya, O.; Morris, A.J.; Hennig, B. Dioxin-like PCB 126 Increases Intestinal Inflammation and Disrupts Gut Microbiota and Metabolic Homeostasis. Environ. Pollut. 2018, 242, 1022-1032. [CrossRef]

11. Stedtfeld, R.D.; Chai, B.; Crawford, R.B.; Stedtfeld, T.M.; Williams, M.R.; Xiangwen, S.; Kuwahara, T.; Cole, J.R.; Kaminski, N.E.; Tiedje, J.M.; et al. Modulatory Influence of Segmented Filamentous Bacteria on Transcriptomic Response of Gnotobiotic Mice Exposed to TCDD. Front. Microbiol. 2017, 8, 1708. [CrossRef]

12. Nault, R.; Fader, K.A.; Lydic, T.A.; Zacharewski, T.R. Lipidomic Evaluation of Aryl Hydrocarbon Receptor-Mediated Hepatic Steatosis in Male and Female Mice Elicited by 2,3,7,8-Tetrachlorodibenzo-p-dioxin. Chem. Res. Toxicol. 2017, 30, 1060-1075. [CrossRef] [PubMed]

13. Nault, R.; Fader, K.A.; Ammendolia, D.A.; Dornbos, P.; Potter, D.; Sharratt, B.; Kumagai, K.; Harkema, J.R.; Lunt, S.Y.; Matthews, J.; et al. Dose-Dependent Metabolic Reprogramming and Differential Gene Expression in TCDD-Elicited Hepatic Fibrosis. Toxicol. Sci. 2016, 154, 253-266. [CrossRef] [PubMed]

14. Fader, K.A.; Nault, R.; Ammendolia, D.A.; Harkema, J.R.; Williams, K.J.; Crawford, R.B.; Kaminski, N.E.; Potter, D.; Sharratt, B.; Zacharewski, T.R. 2,3,7,8-Tetrachlorodibenzo- $p$-Dioxin Alters Lipid Metabolism and Depletes Immune Cell Populations in the Jejunum of C57BL/6 Mice. Toxicol. Sci. 2015, 148, 567-580. [CrossRef] [PubMed]

15. Angrish, M.M.; Dominici, C.Y.; Zacharewski, T.R. TCDD-Elicited Effects on Liver, Serum, and Adipose Lipid Composition in C57BL/6 Mice. Toxicol. Sci. 2013, 131, 108-115. [CrossRef]

16. Katsiki, N.; Mikhailidis, D.P.; Mantzoros, C.S. Non-Alcoholic Fatty Liver Disease and Dyslipidemia: An Update. Metabolism 2016, 65, 1109-1123. [CrossRef]

17. Cholico, G.N.; Fling, R.R.; Zacharewski, N.A.; Fader, K.A.; Nault, R.; Zacharewski, T. Thioesterase Induction by 2,3,7,8Tetrachlorodibenzo- $p$-Dioxin Results in a Futile Cycle That Inhibits Hepatic $\beta$-Oxidation. bioRxiv 2021. [CrossRef]

18. Fernandez-Salguero, P.M.; Hllbert, D.M.; Rudikoff, S.; Ward, J.M.; Gonzalez, F.J. Aryl-Hydrocarbon Receptor-Deficient Mice Are Resistant to 2,3,7,8-Tetrachlorodibenzo-p-Dioxin-Induced Toxicity. Toxicol. Appl. Pharmacol. 1996, 140, 173-179. [CrossRef]

19. Bock, K.W. Aryl Hydrocarbon Receptor (AHR)-Mediated Inflammation and Resolution: Non-Genomic and Genomic Signaling. Biochem. Pharmacol. 2020, 182, 114220. [CrossRef] 
20. Schiering, C.; Wincent, E.; Metidji, A.; Iseppon, A.; Li, Y.; Potocnik, A.J.; Omenetti, S.; Henderson, C.J.; Wolf, C.R.; Nebert, D.W.; et al. Feedback Control of AHR Signalling Regulates Intestinal Immunity. Nature 2017, 542, 242-245. [CrossRef]

21. Grabherr, F.; Grander, C.; Effenberger, M.; Adolph, T.E.; Tilg, H. Gut Dysfunction and Non-Alcoholic Fatty Liver Disease. Front. Endocrinol. 2019, 10, 611. [CrossRef]

22. Leung, C.; Rivera, L.; Furness, J.B.; Angus, P.W. The Role of the Gut Microbiota in NAFLD. Nat. Rev. Gastroenterol. Hepatol. 2016, 13, 412-425. [CrossRef]

23. Tian, J.; Feng, Y.; Fu, H.; Xie, H.Q.; Jiang, J.X.; Zhao, B. The Aryl Hydrocarbon Receptor: A Key Bridging Molecule of External and Internal Chemical Signals. Environ. Sci. Technol. 2015, 49, 9518-9531. [CrossRef]

24. Rothhammer, V.; Quintana, F.J. The Aryl Hydrocarbon Receptor: An Environmental Sensor Integrating Immune Responses in Health and Disease. Nat. Rev. Immunol. 2019, 19, 184-197. [CrossRef]

25. Qiu, J.; Heller, J.J.; Guo, X.; Chen, Z.M.E.; Fish, K.; Fu, Y.X.; Zhou, L. The Aryl Hydrocarbon Receptor Regulates Gut Immunity through Modulation of Innate Lymphoid Cells. Immunity 2012, 36, 92-104. [CrossRef]

26. Zelante, T.; Iannitti, R.G.; Cunha, C.; DeLuca, A.; Giovannini, G.; Pieraccini, G.; Zecchi, R.; D'Angelo, C.; Massi-Benedetti, C.; Fallarino, F; et al. Tryptophan Catabolites from Microbiota Engage Aryl Hydrocarbon Receptor and Balance Mucosal Reactivity via Interleukin-22. Immunity 2013, 39, 372-385. [CrossRef] [PubMed]

27. Murray, I.A.; Nichols, R.G.; Zhang, L.; Patterson, A.D.; Perdew, G.H. Expression of the Aryl Hydrocarbon Receptor Contributes to the Establishment of Intestinal Microbial Community Structure in Mice. Sci. Rep. 2016, 6, 33969. [CrossRef]

28. Lefever, D.E.; Xu, J.; Chen, Y.; Huang, G.; Tamas, N.; Guo, T.L. TCDD Modulation of Gut Microbiome Correlated with Liver and Immune Toxicity in Streptozotocin (STZ)-Induced Hyperglycemic Mice. Toxicol. Appl. Pharmacol. 2016, 304, 48-58. [CrossRef] [PubMed]

29. Neamah, W.H.; Busbee, P.B.; Alghetaa, H.; Abdulla, O.A.; Nagarkatti, M.; Nagarkatti, P. AhR Activation Leads to Alterations in the Gut Microbiome with Consequent Effect on Induction of Myeloid Derived Suppressor Cells in a CXCR2-Dependent Manner. Int. J. Mol. Sci. 2020, 18, 9613. [CrossRef] [PubMed]

30. Stedtfeld, R.D.; Brett Sallach, J.; Crawford, R.B.; Stedtfeld, T.M.; Williams, M.R.; Waseem, H.; Johnston, C.T.; Li, H.; Teppen, B.J.; Kaminski, N.E.; et al. TCDD Administered on Activated Carbon Eliminates Bioavailability and Subsequent Shifts to a Key Murine Gut Commensal. Appl. Microbiol. Biotechnol. 2017, 101, 7409-7415. [CrossRef]

31. Shao, J.-W.; Ge, T.-T.; Chen, S.-Z.; Wang, G.; Yang, Q.; Huang, C.-H.; Xu, L.-C.; Chen, Z. Role of Bile Acids in Liver Diseases Mediated by the Gut Microbiome. World J. Gastroenterol. 2021, 27, 3010-3021. [CrossRef] [PubMed]

32. Marion, S.; Desharnais, L.; Studer, N.; Dong, Y.; Notter, M.D.; Poudel, S.; Menin, L.; Janowczyk, A.; Hettich, R.L.; Hapfelmeier, S.; et al. Biogeography of Microbial Bile Acid Transformations along the Murine Gut. J. Lipid Res. 2020, 61, 1450-1463. [CrossRef] [PubMed]

33. Foley, M.H.; O'Flaherty, S.; Allen, G.; Rivera, A.J.; Stewart, A.K.; Barrangou, R.; Theriot, C.M. Lactobacillus Bile Salt Hydrolase Substrate Specificity Governs Bacterial Fitness and Host Colonization. Proc. Natl. Acad. Sci. USA 2021, 118, e2017709118. [CrossRef]

34. Matsubara, T.; Li, F.; Gonzalez, F.J. FXR Signaling in the Enterohepatic System. Mol. Cell. Endocrinol. 2013, 368, 17-29. [CrossRef] [PubMed]

35. Shapiro, H.; Kolodziejczyk, A.A.; Halstuch, D.; Elinav, E. Bile Acids in Glucose Metabolism in Health and Disease. J. Exp. Med. 2018, 215, 383-396. [CrossRef]

36. Pols, T.W.H.; Noriega, L.G.; Nomura, M.; Auwerx, J.; Schoonjans, K. The Bile Acid Membrane Receptor TGR5: A Valuable Metabolic Target. Dig. Dis 2011, 29, 37-44. [CrossRef]

37. Fiorucci, S.; Mencarelli, A.; Palladino, G.; Cipriani, S. Bile-Acid-Activated Receptors: Targeting TGR5 and Farnesoid-X-Receptor in Lipid and Glucose Disorders. Trends Pharmacol. Sci. 2009, 30, 570-580. [CrossRef]

38. O'Flaherty, S.; Briner Crawley, A.; Theriot, C.M.; Barrangou, R. The Lactobacillus Bile Salt Hydrolase Repertoire Reveals NicheSpecific Adaptation. mSphere 2018, 3, e00140-18. [CrossRef] [PubMed]

39. Shao, L.; Ling, Z.; Chen, D.; Liu, Y.; Yang, F.; Li, L. Disorganized Gut Microbiome Contributed to Liver Cirrhosis Progression: A Meta-Omics-Based Study. Front. Microbiol. 2018, 9, 3166. [CrossRef]

40. Fazili, T.; Riddell, S.; Kiska, D.; Endy, T.; Giurgea, L.; Sharngoe, C.; Javaid, W. Streptococcus Anginosus Group Bacterial Infections. Am. J. Med. Sci. 2017, 354, 257-261. [CrossRef]

41. Workman, S.D.; Strynadka, N.C.J. A Slippery Scaffold: Synthesis and Recycling of the Bacterial Cell Wall Carrier Lipid. J. Mol. Biol. 2020, 432, 4964-4982. [CrossRef]

42. Johnston, J.M.; Bulloch, E.M. Advances in Menaquinone Biosynthesis: Sublocalisation and Allosteric Regulation. Curr. Opin. Struct. Biol. 2020, 65, 33-41. [CrossRef] [PubMed]

43. Hernández, S.B.; Cava, F.; Pucciarelli, M.G.; García-del Portillo, F.; de Pedro, M.A.; Casadesús, J. Bile-Induced Peptidoglycan Remodelling in Salmonella enterica: Bile-Induced Peptidoglycan Remodelling. Environ. Microbiol. 2015, 17, 1081-1089. [CrossRef] [PubMed]

44. Ravcheev, D.A.; Thiele, I. Genomic Analysis of the Human Gut Microbiome Suggests Novel Enzymes Involved in Quinone Biosynthesis. Front. Microbiol. 2016, 7, 128. [CrossRef] [PubMed] 
45. Aron-Wisnewsky, J.; Vigliotti, C.; Witjes, J.; Le, P.; Holleboom, A.G.; Verheij, J.; Nieuwdorp, M.; Clément, K. Gut Microbiota and Human NAFLD: Disentangling Microbial Signatures from Metabolic Disorders. Nat. Rev. Gastroenterol. Hepatol. 2020, 17, $279-297$. [CrossRef] [PubMed]

46. Qiao, Y.; Sun, J.; Xia, S.; Li, L.; Li, Y.; Wang, P.; Shi, Y.; Le, G. Effects of Different Lactobacillus Reuteri on Inflammatory and Fat Storage in High-Fat Diet-Induced Obesity Mice Model. J. Funct. Foods 2015, 14, 424-434. [CrossRef]

47. Ting, W.-J.; Kuo, W.-W.; Hsieh, D.; Yeh, Y.-L.; Day, C.-H.; Chen, Y.-H.; Chen, R.-J.; Padma, V.; Chen, Y.-H.; Huang, C.-Y. Heat Killed Lactobacillus Reuteri GMNL-263 Reduces Fibrosis Effects on the Liver and Heart in High Fat Diet-Hamsters via TGF- $\beta$ Suppression. Int. J. Mol. Sci. 2015, 16, 25881-25896. [CrossRef] [PubMed]

48. Andreasen, A.S.; Larsen, N.; Pedersen-Skovsgaard, T.; Berg, R.M.G.; Møller, K.; Svendsen, K.D.; Jakobsen, M.; Pedersen, B.K. Effects of Lactobacillus acidophilus NCFM on Insulin Sensitivity and the Systemic Inflammatory Response in Human Subjects. Br. J. Nutr. 2010, 104, 1831-1838. [CrossRef]

49. Khare, A.; Gaur, S. Cholesterol-Lowering Effects of Lactobacillus Species. Curr. Microbiol. 2020, 77, 638-644. [CrossRef] [PubMed]

50. Roh, Y.S.; Cho, A.; Cha, Y.-S.; Oh, S.-H.; Lim, C.W.; Kim, B. Lactobacillus Aggravate Bile Duct Ligation-Induced Liver Inflammation and Fibrosis in Mice. Toxicol. Res. 2018, 34, 241-247. [CrossRef]

51. Martoni, C.J.; Labbé, A.; Ganopolsky, J.G.; Prakash, S.; Jones, M.L. Changes in Bile Acids, FGF-19 and Sterol Absorption in Response to Bile Salt Hydrolase Active L. reuteri NCIMB 30242. Gut Microbes 2015, 6, 57-65. [CrossRef] [PubMed]

52. Kumar, R.; Grover, S.; Batish, V.K. Hypocholesterolaemic Effect of Dietary Inclusion of Two Putative Probiotic Bile Salt HydrolaseProducing Lactobacillus plantarum Strains in Sprague-Dawley Rats. Br. J. Nutr. 2011, 105, 561-573. [CrossRef] [PubMed]

53. Lee, N.Y.; Shin, M.J.; Youn, G.S.; Yoon, S.J.; Choi, Y.R.; Kim, H.S.; Gupta, H.; Han, S.H.; Kim, B.K.; Lee, D.Y.; et al. Lactobacillus Attenuates Progression of Nonalcoholic Fatty Liver Disease by Lowering Cholesterol and Steatosis. Clin. Mol. Hepatol. 2021, 27, 110-124. [CrossRef]

54. Kemis, J.H.; Linke, V.; Barrett, K.L.; Boehm, F.J.; Traeger, L.L.; Keller, M.P.; Rabaglia, M.E.; Schueler, K.L.; Stapleton, D.S.; Gatti, D.M.; et al. Genetic Determinants of Gut Microbiota Composition and Bile Acid Profiles in Mice. PLoS Genet. 2019, 15, e1008073. [CrossRef] [PubMed]

55. Fung, T.C.; Vuong, H.E.; Luna, C.D.G.; Pronovost, G.N.; Aleksandrova, A.A.; Riley, N.G.; Vavilina, A.; McGinn, J.; Rendon, T.; Forrest, L.R.; et al. Intestinal Serotonin and Fluoxetine Exposure Modulate Bacterial Colonization in the Gut. Nat. Microbiol. 2019, 4, 2064-2073. [CrossRef]

56. Tannock, G.W.; Dashkevicz, M.P.; Feighner, S.D. Lactobacilli and Bile Salt Hydrolase in the Murine Intestinal Tract. Appl. Environ. Microbiol. 1989, 55, 1848-1851. [CrossRef]

57. Winston, J.A.; Rivera, A.; Cai, J.; Patterson, A.D.; Theriot, C.M. Secondary Bile Acid Ursodeoxycholic Acid Alters Weight, the Gut Microbiota, and the Bile Acid Pool in Conventional Mice. PLoS ONE 2021, 16, e0246161. [CrossRef]

58. Chae, J.P.; Valeriano, V.D.; Kim, G.-B.; Kang, D.-K. Molecular Cloning, Characterization and Comparison of Bile Salt Hydrolases from Lactobacillus johnsonii PF01. J. Appl. Microbiol. 2013, 114, 121-133. [CrossRef]

59. De Smet, I.; Van Hoorde, L.; Vande Woestyne, M.; Christiaens, H.; Verstraete, W. Significance of Bile Salt Hydrolytic Activities of Lactobacilli. J. Appl. Bacteriol. 1995, 79, 292-301. [CrossRef]

60. Bustos, A.Y.; Saavedra, L.; de Valdez, G.F.; Raya, R.R.; Taranto, M.P. Relationship between Bile Salt Hydrolase Activity, Changes in the Internal PH and Tolerance to Bile Acids in Lactic Acid Bacteria. Biotechnol. Lett. 2012, 34, 1511-1518. [CrossRef]

61. Sato, H.; Macchiarulo, A.; Thomas, C.; Gioiello, A.; Une, M.; Hofmann, A.F.; Saladin, R.; Schoonjans, K.; Pellicciari, R.; Auwerx, J. Novel Potent and Selective Bile Acid Derivatives as TGR5 Agonists: Biological Screening, Structure-Activity Relationships, and Molecular Modeling Studies. J. Med. Chem. 2008, 51, 1831-1841. [CrossRef]

62. Wahlström, A.; Sayin, S.I.; Marschall, H.-U.; Bäckhed, F. Intestinal Crosstalk between Bile Acids and Microbiota and Its Impact on Host Metabolism. Cell Metab. 2016, 24, 41-50. [CrossRef]

63. Vranová, E.; Coman, D.; Gruissem, W. Network Analysis of the MVA and MEP Pathways for Isoprenoid Synthesis. Annu. Rev. Plant Biol. 2013, 64, 665-700. [CrossRef] [PubMed]

64. Stacchiotti, V.; Rezzi, S.; Eggersdorfer, M.; Galli, F. Metabolic and Functional Interplay between Gut Microbiota and Fat-Soluble Vitamins. Crit. Rev. Food Sci. Nutr. 2020, 61, 3211-3232. [CrossRef] [PubMed]

65. Brooijmans, R.; Smit, B.; Santos, F.; van Riel, J.; de Vos, W.M.; Hugenholtz, J. Heme and Menaquinone Induced Electron Transport in Lactic Acid Bacteria. Microb Cell Fact. 2009, 8, 28. [CrossRef] [PubMed]

66. Ianniello, R.G.; Zheng, J.; Zotta, T.; Ricciardi, A.; Gänzle, M.G. Biochemical Analysis of Respiratory Metabolism in the Heterofermentative Lactobacillus spicheri and Lactobacillus reuteri. J. Appl. Microbiol. 2015, 119, 763-775. [CrossRef]

67. Plichta, D.R.; Somani, J.; Pichaud, M.; Wallace, Z.S.; Fernandes, A.D.; Perugino, C.A.; Lähdesmäki, H.; Stone, J.H.; Vlamakis, H.; Chung, D.C.; et al. Congruent Microbiome Signatures in Fibrosis-Prone Autoimmune Diseases: IgG4-Related Disease and Systemic Sclerosis. Genome Med. 2021, 13, 35. [CrossRef]

68. Quinn, R.A.; Whiteson, K.; Lim, Y.W.; Zhao, J.; Conrad, D.; LiPuma, J.J.; Rohwer, F.; Widder, S. Ecological Networking of Cystic Fibrosis Lung Infections. NPJ Biofilms Microbiomes 2016, 2, 4. [CrossRef]

69. Chu, N.-N.; Chen, W.-L.; Xu, H.-R.; Li, X.-N. Pharmacokinetics and Safety of Ezetimibe/Simvastatin Combination Tablet: An Open-Label, Single-Dose Study in Healthy Chinese Subjects. Clin. Drug Investig. 2012, 32, 791-798. [CrossRef]

70. Ashrafizadeh, M.; Ahmadi, Z. Effects of Statins on Gut Microbiota (Microbiome). Rev. Clin. Med. 2019, 6, 5. 
71. Whitaker, E.; Alshammari, A. Bacteriostatic Effect of Simvastatin on Selected Oral Streptococci in Vitro. Contemp. Clin. Dent. 2017, 8, 59. [CrossRef]

72. Wu, S.; Jiang, P.; Zhao, X.-M.; Chen, W.-H. Treatment Regimens May Compromise Gut-Microbiome-Derived Signatures for Liver Cirrhosis. Cell Metab. 2021, 33, 455-456. [CrossRef]

73. Loomba, R.; Seguritan, V.; Li, W.; Long, T.; Klitgord, N.; Bhatt, A.; Dulai, P.S.; Caussy, C.; Bettencourt, R.; Highlander, S.K.; et al Gut Microbiome-Based Metagenomic Signature for Non-Invasive Detection of Advanced Fibrosis in Human Nonalcoholic Fatty Liver Disease. Cell Metab. 2017, 25, 1054-1062.e5. [CrossRef]

74. McCoy, K.D.; Ohland, C.L. Innate Responses to Gut Microbiota; Critical Assessment of the Necessary Experimental Controls. Curr. Opin. Microbiol. 2021, 59, 34-41. [CrossRef] [PubMed]

75. Thaiss, C.A.; Zeevi, D.; Levy, M.; Zilberman-Schapira, G.; Suez, J.; Tengeler, A.C.; Abramson, L.; Katz, M.N.; Korem, T.; Zmora, N.; et al. Transkingdom Control of Microbiota Diurnal Oscillations Promotes Metabolic Homeostasis. Cell 2014, 159, 514-529. [CrossRef] [PubMed]

76. Fader, K.A.; Nault, R.; Doskey, C.M.; Fling, R.R.; Zacharewski, T.R. 2,3,7,8-Tetrachlorodibenzo-p-Dioxin Abolishes Circadian Regulation of Hepatic Metabolic Activity in Mice. Sci. Rep. 2019, 9, 6514. [CrossRef] [PubMed]

77. Nault, R.; Fader, K.A.; Harkema, J.R.; Zacharewski, T. Loss of Liver-Specific and Sexually Dimorphic Gene Expression by Aryl Hydrocarbon Receptor Activation in C57BL/6 Mice. PLoS ONE 2017, 12, e0184842. [CrossRef]

78. Fling, R.R.; Doskey, C.M.; Fader, K.A.; Nault, R.; Zacharewski, T.R. 2,3,7,8-Tetrachlorodibenzo- $p$-Dioxin (TCDD) Dysregulates Hepatic One Carbon Metabolism during the Progression of Steatosis to Steatohepatitis with Fibrosis in Mice. Sci. Rep. 2020, 10, 14831. [CrossRef]

79. Lee, G.; You, H.J.; Bajaj, J.S.; Joo, S.K.; Yu, J.; Park, S.; Kang, H.; Park, J.H.; Kim, J.H.; Lee, D.H.; et al. Distinct Signatures of Gut Microbiome and Metabolites Associated with Significant Fibrosis in Non-Obese NAFLD. Nat. Commun. 2020, 11, 4982. [CrossRef]

80. Jiao, N.; Baker, S.S.; Chapa-Rodriguez, A.; Liu, W.; Nugent, C.A.; Tsompana, M.; Mastrandrea, L.; Buck, M.J.; Baker, R.D.; Genco, R.J.; et al. Suppressed Hepatic Bile Acid Signalling despite Elevated Production of Primary and Secondary Bile Acids in NAFLD. Gut 2018, 67, 1881-1891. [CrossRef]

81. Aranha, M.M.; Cortez-Pinto, H.; Costa, A.; da Silva, I.B.M.; Camilo, M.E.; de Moura, M.C.; Rodrigues, C.M.P. Bile Acid Levels Are Increased in the Liver of Patients with Steatohepatitis. Eur. J. Gastroenterol. Hepatol. 2008, 20, 519-525. [CrossRef] [PubMed]

82. Kakiyama, G.; Pandak, W.M.; Gillevet, P.M.; Hylemon, P.B.; Heuman, D.M.; Daita, K.; Takei, H.; Muto, A.; Nittono, H.; Ridlon, J.M.; et al. Modulation of the Fecal Bile Acid Profile by Gut Microbiota in Cirrhosis. J. Hepatol. 2013, 58, 949-955. [CrossRef] [PubMed]

83. Jiao, N.; Loomba, R.; Yang, Z.-H.; Wu, D.; Fang, S.; Bettencourt, R.; Lan, P.; Zhu, R.; Zhu, L. Alterations in Bile Acid Metabolizing Gut Microbiota and Specific Bile Acid Genes as a Precision Medicine to Subclassify NAFLD. Physiol. Genom. 2020, 53, 336-348. [CrossRef] [PubMed]

84. Plant, L.; Conway, P. Association of Lactobacillus spp. with Peyer's Patches in Mice. Clin. Diagn Lab. Immunol. 2001, 8, 320-324. [CrossRef]

85. Wang, P.; Li, Y.; Xiao, H.; Shi, Y.; Le, G.; Sun, J. Isolation of Lactobacillus reuteri from Peyer's Patches and Their Effects on SIgA Production and Gut Microbiota Diversity. Mol. Nutr. Food Res. 2016, 60, 2020-2030. [CrossRef] [PubMed]

86. Fader, K.A.; Nault, R.; Raehtz, S.; McCabe, L.R.; Zacharewski, T.R. 2,3,7,8-Tetrachlorodibenzo- $p$-Dioxin Dose-Dependently Increases Bone Mass and Decreases Marrow Adiposity in Juvenile Mice. Toxicol. Appl. Pharmacol. 2018, 348, 85-98. [CrossRef]

87. Collins, F.L.; Irwin, R.; Bierhalter, H.; Schepper, J.; Britton, R.A.; Parameswaran, N.; McCabe, L.R. Lactobacillus Reuteri 6475 Increases Bone Density in Intact Females Only under an Inflammatory Setting. PLoS ONE 2016, 11, e0153180. [CrossRef]

88. Langmead, B.; Salzberg, S.L. Fast Gapped-Read Alignment with Bowtie 2. Nat. Methods 2012, 9, 357-359. [CrossRef]

89. Danecek, P.; Bonfield, J.K.; Liddle, J.; Marshall, J.; Ohan, V.; Pollard, M.O.; Whitwham, A.; Keane, T.; McCarthy, S.A.; Davies, R.M.; et al. Twelve Years of SAMtools and BCFtools. GigaScience 2021, 10, giab008. [CrossRef]

90. Quinlan, A.R.; Hall, I.M. BEDTools: A Flexible Suite of Utilities for Comparing Genomic Features. Bioinformatics 2010, 26, 841-842. [CrossRef]

91. Mallick, H.; Rahnavard, A.; McIver, L.J.; Ma, S.; Zhang, Y.; Nguyen, L.H.; Tickle, T.L.; Weingart, G.; Ren, B.; Schwager, E.H.; et al. Multivariable Association Discovery in Population-Scale Meta-Omics Studies. Biorxiv 2021. [CrossRef]

92. Love, M.I.; Huber, W.; Anders, S. Moderated Estimation of Fold Change and Dispersion for RNA-Seq Data with DESeq2. Genome Biol. 2014, 15, 550. [CrossRef] [PubMed]

93. Franzosa, E.A.; Morgan, X.C.; Segata, N.; Waldron, L.; Reyes, J.; Earl, A.M.; Giannoukos, G.; Boylan, M.R.; Ciulla, D.; Gevers, D.; et al. Relating the Metatranscriptome and Metagenome of the Human Gut. Proc. Natl. Acad. Sci. USA 2014, 111, E2329-E2338. [CrossRef] [PubMed]

94. Wang, Q.; Fish, J.A.; Gilman, M.; Sun, Y.; Brown, C.T.; Tiedje, J.M.; Cole, J.R. Xander: Employing a Novel Method for Efficient Gene-Targeted Metagenomic Assembly. Microbiome 2015, 3, 32. [CrossRef] [PubMed]

95. Fish, J.A.; Chai, B.; Wang, Q.; Sun, Y.; Brown, C.T.; Tiedje, J.M.; Cole, J.R. FunGene: The Functional Gene Pipeline and Repository. Front. Microbiol. 2013, 4, 291. [CrossRef] 